JOURNAL OF THE

AMERICAN MATHEMATICAL SOCIETY

Volume 24, Number 4, October 2011, Pages 969-998

S 0894-0347(2011)00701-7

Article electronically published on April 6, 2011

\title{
HALL ALGEBRAS AND CURVE-COUNTING INVARIANTS
}

\author{
TOM BRIDGELAND
}

\section{INTRODUCTION}

In this paper we use Joyce's theory of motivic Hall algebras to prove some basic properties of Donaldson-Thomas (DT) curve-counting invariants on Calabi-Yau threefolds. We prove that the reduced DT invariants coincide with the stable pair invariants introduced by Pandharipande and Thomas and that the generating functions for these invariants are Laurent expansions of rational functions. Similar results have been obtained by Toda; we discuss the relationship with his work below.

The Hall algebra approach to DT invariants relies on a fundamental result of Behrend. Recall [1] that Behrend associates to any scheme $S$ of finite type over $\mathbb{C}$ a constructible function

$$
\nu_{S}: S \rightarrow \mathbb{Z}
$$

with the property that if $S$ is a proper moduli scheme with a symmetric obstruction theory, then the associated virtual counting invariant

$$
\#_{\text {vir }}(S):=\int_{[S]^{\mathrm{vir}}} 1
$$

coincides with the weighted Euler characteristic

$$
\chi\left(S, \nu_{S}\right):=\sum_{n \in \mathbb{Z}} n \chi\left(\nu_{S}^{-1}(n)\right) .
$$

This means that the virtual count can be computed by motivic techniques involving decomposing $S$ into a disjoint union of locally closed subschemes.

Suppose that $M$ is a smooth projective Calabi-Yau threefold over $\mathbb{C}$. We include in this assumption the condition that

$$
H^{1}\left(M, \mathcal{O}_{M}\right)=0 .
$$

Let $N_{1}(M)$ denote the abelian group of curves in $M$ up to numerical equivalence. It is a free abelian group of finite rank. Set

$$
N_{\leqslant 1}(M)=N_{1}(M) \oplus \mathbb{Z} .
$$

A coherent sheaf $E$ on $M$ supported in dimension $\leqslant 1$ has a Chern character

$$
\operatorname{ch}(E)=\left(\operatorname{ch}_{2}(E), \operatorname{ch}_{3}(E)\right)=(\beta, n) \in N_{\leqslant 1}(M) .
$$

Given a class $(\beta, n) \in N_{\leqslant 1}(M)$, there is an open and closed subset of the Hilbert scheme

$$
\operatorname{Hilb}_{M}(\beta, n) \subset \operatorname{Hilb}_{M}
$$

Received by the editors February 25, 2010 and, in revised form, March 9, 2011

2010 Mathematics Subject Classification. Primary 14N35, 14D23.

(C)2011 American Mathematical Society 
parameterizing epimorphisms of coherent sheaves

$$
f: \mathcal{O}_{M} \rightarrow E
$$

with $E$ of Chern character $(\beta, n)$. The corresponding DT curve-counting invariant is

$$
\operatorname{DT}(\beta, n)=\#_{\text {vir }}\left(\operatorname{Hilb}_{M}(\beta, n)\right) \in \mathbb{Z} .
$$

For a fixed curve class $\beta \in N_{1}(M)$ the scheme $\operatorname{Hilb}_{M}(\beta, n)$ is known to be empty for $n \ll 0$, so the generating function

$$
\operatorname{DT}_{\beta}(q)=\sum_{n \in \mathbb{Z}} \operatorname{DT}(\beta, n) q^{n}
$$

is a Laurent series.

In the case $\beta=0$ the scheme $\operatorname{Hilb}_{M}(\beta, n)$ parameterizes zero-dimensional subschemes of $M$ of length $n$, and the corresponding generating function $\operatorname{DT}_{0}(q)$ is known [2, 19, 20]. It is the Taylor expansion of the function

$$
M(-q)^{\chi(M)}
$$

where $\chi(M)$ is the topological Euler characteristic of $M$, and the MacMahon function

$$
M(q)=\prod_{k \geqslant 1}\left(1-q^{k}\right)^{-k}
$$

is the generating function for three-dimensional partitions. Following [21], one defines the reduced DT generating function to be the quotient

$$
\mathrm{DT}_{\beta}^{\prime}(q)=\mathrm{DT}_{\beta}(q) / \mathrm{DT}_{0}(q)
$$

It is a Laurent series with integral coefficients.

Pandharipande and Thomas [23] introduced new curve-counting invariants by considering stable pairs. These are maps of coherent sheaves

$$
f: \mathcal{O}_{M} \rightarrow E
$$

such that $E$ is supported in dimension one and such that

(a) $E$ has no zero-dimensional subsheaves,

(b) the cokernel of $f$ is zero-dimensional.

In fact, as we prove in Lemma 2.3 below, such maps can be though of as epimorphisms in the heart of a certain perverse t-structure on the derived category of $M$.

It was proved in 23 that there is a fine moduli scheme parameterizing stable pairs, which we will denote by $\operatorname{Hilb}_{M}^{\sharp}$. For each class $(\beta, n) \in N_{\leqslant 1}(M)$ there is an open and closed subscheme

$$
\operatorname{Hilb}_{M}^{\sharp}(\beta, n) \subset \operatorname{Hilb}_{M}^{\sharp}
$$

parameterizing stable pairs with $E$ of Chern character $(\beta, n)$. The corresponding PT invariant is

$$
\operatorname{PT}(\beta, n)=\#_{\operatorname{vir}}\left(\operatorname{Hilb}_{M}^{\sharp}(\beta, n)\right) \in \mathbb{Z} .
$$

Once again, for fixed $\beta \in N_{1}(M)$ the generating function

$$
\operatorname{PT}_{\beta}(q)=\sum_{n \in \mathbb{Z}} \operatorname{PT}(\beta, n) q^{n}
$$

is a Laurent series. Note that in the case $\beta=0$ we have $\mathrm{PT}_{0} \equiv 1$. 
Our main result is as follows.

Theorem 1.1. Let $M$ be a smooth projective Calabi-Yau threefold. Then for each class $\beta \in N_{1}(M)$

(a) there is an equality of Laurent series

$$
\mathrm{DT}_{\beta}^{\prime}(q)=\operatorname{PT}_{\beta}(q)
$$

(b) the series $\mathrm{DT}_{\beta}^{\prime}(q)$ is the Laurent expansion of a rational function in $q$, invariant under the transformation $q \leftrightarrow q^{-1}$.

The first part of Theorem 1.1 (the DT/PT correspondence) was conjectured by Pandharipande and Thomas [23, Conj. 3.3]. The second part was conjectured earlier by Maulik, Nekrasov, Okounkov, and Pandharipande [21, Conj. 2] and is a crucial ingredient in the conjectural DT/GW correspondence [21, Conj. 3].

There is another version of Theorem 1.1 involving unweighted Euler characteristic invariants defined without a Behrend function. This was first proved by Toda 27, 28. Our argument gives a second proof of this result, and Stoppa and Thomas 25] have given a third (and simpler) proof, which even extends to the non-CalabiYau case.

The approach to Theorem 1.1 that we present here owes a lot to Toda's work [27], and we have used ideas from his paper in several places. In fact Toda's argument could be modified to give a proof of Theorem 1.1 if one could establish a certain identity involving Behrend weights for objects of the derived category of $M$. The corresponding identity for coherent sheaves was established by Joyce and Song [16] and plays a crucial role in this paper.

The main difference between our approach and that of [27] is that Toda views the scheme $\operatorname{Hilb}_{M}(\beta, n)$ as parameterizing stable rank 1 ideal sheaves on $M$, whereas we consider it as parameterizing sheaves supported in dimension $\leqslant 1$ together with the framing data of an epimorphism from $\mathcal{O}_{M}$.

Notation. By a stack we mean an algebraic (Artin) stack. All schemes and stacks will be locally of finite type over $\mathbb{C}$. Throughout $M$ will be a smooth complex projective threefold with trivial canonical bundle and satisfying $H^{1}\left(M, \mathcal{O}_{M}\right)=0$. We write $\mathcal{A}=\operatorname{Coh}(M)$ for the abelian category of coherent sheaves on $M$ and denote by $\mathcal{M}$ the moduli stack of objects of $\mathcal{A}$. We frequently use the same letter for an open substack of $\mathcal{M}$ and the corresponding full subcategory of $\mathcal{A}$. One other minor abuse of notation: if $f: T \rightarrow S$ is a morphism of schemes and $E$ is a sheaf on $S \times M$, we use the shorthand $f^{*}(E)$ for the pullback to $T \times M$ rather than the more correct $\left(f \times \operatorname{id}_{M}\right)^{*}(E)$.

\section{Preliminaries}

We begin by assembling some basic facts that will be needed later.

2.1. Curve classes. We denote by $N_{1}(M)$ the abelian group of cycles of dimension 1 on $M$ modulo numerical equivalence. It is a free abelian group of finite rank 6 , Ex. 19.1.4]. An element $\beta \in N_{1}(M)$ is effective, written $\beta \geqslant 0$, if it is zero or the class of a curve.

Lemma 2.1. An element $\beta \in N_{1}(M)$ has only finitely many decompositions of the form $\beta=\beta_{1}+\beta_{2}$ with $\beta_{i} \geqslant 0$. 
Proof. This follows immediately from [17, Cor. 1.19].

We denote by $N(M)$ the numerical Grothendieck group of $M$. It is the quotient of the Grothendieck group $K(M)$ by the kernel of the Euler form. There is a subgroup

$$
N_{\leqslant 1}(M) \subset N(M)
$$

generated by classes of sheaves supported in dimension at most 1 .

Lemma 2.2. The Chern character induces an isomorphism

$$
\operatorname{ch}=\left(\operatorname{ch}_{2}, \operatorname{ch}_{3}\right): N_{\leqslant 1}(M) \longrightarrow N_{1}(M) \oplus \mathbb{Z} .
$$

Proof. The Chern character defines a map to the Chow ring

$$
\operatorname{ch}: K(M) \rightarrow A^{*}(M) \otimes \mathbb{Q} \text {. }
$$

Let $K_{\leqslant 1}(M) \subset K(M)$ be the subgroup spanned by classes of sheaves supported in dimension at most 1 . The Chern character of such a sheaf is equal to its Chern class for dimension reasons and in particular is integral. Thus for any $\alpha \in K_{\leqslant 1}(M)$

$$
\operatorname{ch}(\alpha)=\left(\operatorname{ch}_{2}(\alpha), \operatorname{ch}_{3}(\alpha)\right) \in A_{1}(M) \oplus \mathbb{Z} .
$$

By the Riemann-Roch theorem, the Euler form on $K(M)$ is given by the formula

$$
\chi(\alpha, \beta)=\left[\operatorname{ch}\left(\alpha^{\vee}\right) \cdot \operatorname{ch}(\beta) \cdot \operatorname{td}(M)\right]_{3},
$$

where $\operatorname{td}(M)$ is the Todd class of $M$. It follows that an element $\alpha \in K_{\leqslant 1}(M)$ is numerically trivial precisely if $\operatorname{ch}_{3}(\alpha)=0$ and $\operatorname{ch}_{2}(\alpha) \cdot D=0$ for any divisor $D$. Thus we get an injective map

$$
\operatorname{ch}=\left(\operatorname{ch}_{2}, \operatorname{ch}_{3}\right): N_{\leqslant 1}(M) \rightarrow N_{1}(M) \oplus \mathbb{Z} .
$$

Since $\mathcal{O}_{C}(m)$ maps to $\left([C], \operatorname{ch}_{3}\left(\mathcal{O}_{C}\right)+m\right)$, this map is also surjective.

There is an effective cone

$$
\Delta \subset N_{\leqslant 1}(M)
$$

consisting of classes of sheaves. From now on we shall use the isomorphism of Lemma 2.2 to identify $N_{\leqslant 1}(M)$ with the group $N_{1}(M) \oplus \mathbb{Z}$. Under this identification the effective cone becomes

$$
\Delta=\left\{(\beta, n) \in N_{1}(M) \oplus \mathbb{Z}: \beta>0 \text { or } \beta=0 \text { and } n \geqslant 0\right\} .
$$

Note also that the Euler form $\chi(-,-)$ on $N(M)$ vanishes when restricted to $N_{\leqslant 1}(M)$ for dimension reasons. This trivial observation will be very important in what follows.

2.2. Stable pairs. Define a full subcategory

$$
\mathcal{P}=\operatorname{Coh}_{0}(M) \subset \mathcal{A}=\operatorname{Coh}(M)
$$

consisting of sheaves supported in dimension 0 . Let $\mathcal{Q}$ be the full subcategory

$$
\mathcal{Q}=\{E \in \mathcal{A}: \operatorname{Hom}(P, E)=0 \text { for all } P \in \mathcal{P}\} .
$$

These subcategories $(\mathcal{P}, \mathcal{Q})$ form a torsion pair:

(a) If $P \in \mathcal{P}$ and $Q \in \mathcal{Q}$, then $\operatorname{Hom}_{\mathcal{A}}(P, Q)=0$.

(b) Every object $E \in \mathcal{A}$ fits into a short exact sequence

$$
0 \longrightarrow P \longrightarrow E \longrightarrow Q \longrightarrow 0
$$

with $P \in \mathcal{P}$ and $Q \in \mathcal{Q}$. 
Note that it follows from (a) that the short exact sequence appearing in (b) is unique up to a unique isomorphism.

We can define a t-structure on $D(\mathcal{A})$ by tilting the standard t-structure with respect to this torsion pair [9]. The resulting t-structure has heart

$$
\mathcal{A}^{\sharp}=\left\{E \in D(\mathcal{A}): H^{0}(E) \in \mathcal{Q}, H^{1}(E) \in \mathcal{P}, H^{i}(E)=0 \text { for } i \notin\{0,1\}\right\} .
$$

Note that $\mathcal{Q}=\mathcal{A} \cap \mathcal{A}^{\sharp}$ and, in particular, $\mathcal{O}_{M} \in \mathcal{A}^{\sharp}$.

Lemma 2.3. (a) If $f: \mathcal{O}_{M} \rightarrow E$ is an epimorphism in $\mathcal{A}^{\sharp}$, then $E \in \mathcal{Q}$, and considering $f$ as a map in $\mathcal{A}$, one has coker $(f) \in \mathcal{P}$.

(b) If $f: \mathcal{O}_{M} \rightarrow E$ is a morphism in $\mathcal{A}$ with $E \in \mathcal{Q}$ and $\operatorname{coker}(f) \in \mathcal{P}$, then $f$ is an epimorphism in $\mathcal{A}^{\sharp}$.

Proof. If $f$ is an epimorphism in $\mathcal{A}^{\sharp}$, then there is a triangle

$$
K \longrightarrow \mathcal{O}_{M} \stackrel{f}{\longrightarrow} E \longrightarrow K[1]
$$

with $K \in \mathcal{A}^{\sharp}$. Applying the long exact sequence in cohomology gives

$$
0 \longrightarrow H^{0}(K) \longrightarrow \mathcal{O}_{M} \stackrel{f}{\longrightarrow} H^{0}(E) \longrightarrow H^{1}(K) \longrightarrow 0 .
$$

It follows that $E=H^{0}(E) \in \mathcal{Q}$ and $\operatorname{coker}(f)=H^{1}(K) \in \mathcal{P}$.

For (b) put the map $f$ in a triangle of the form (1) and consider the associated long exact sequence (2). Then $H^{0}(K)$ is a subsheaf of $\mathcal{O}_{M}$ and hence an object of $\mathcal{Q}$, and

$$
H^{1}(K)=\operatorname{coker}(f) \in \mathcal{P} .
$$

Therefore $K \in \mathcal{A}^{\sharp}$, the triangle is a short exact sequence in $\mathcal{A}^{\sharp}$, and hence $f$ is an epimorphism in $\mathcal{A}^{\sharp}$.

Thus a stable pair in the sense of [23] is precisely an epimorphism in $\mathcal{A}^{\sharp}$ whose image is supported in dimension $\leqslant 1$.

2.3. The stack of framed sheaves. We write $\mathcal{M}$ for the stack of coherent sheaves on $M$. It is an algebraic stack, locally of finite type over $\mathbb{C}$. There is another stack $\mathcal{M}(\mathcal{O})$ with a morphism

$$
q: \mathcal{M}(\mathcal{O}) \rightarrow \mathcal{M}
$$

parameterizing coherent sheaves equipped with a section. More precisely, the objects of $\mathcal{M}(\mathcal{O})$ lying over a scheme $S$ are pairs $(E, \gamma)$ consisting of an $S$-flat coherent sheaf $E$ on $S \times M$ together with a section

$$
\gamma: \mathcal{O}_{S \times M} \rightarrow E .
$$

Given a morphism of schemes $f: T \rightarrow S$ and an object $(F, \delta)$ lying over $T$, a morphism $\theta:(F, \delta) \rightarrow(E, \gamma)$ lying over $f$ is an isomorphism

$$
\theta: f^{*}(E) \rightarrow F
$$

such that the diagram

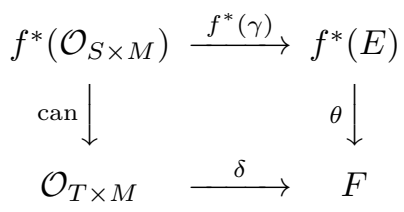


commutes. Here we have taken the usual step of choosing, for each morphism of schemes, a pullback of each coherent sheaf on its target. The symbol can denotes the canonical isomorphism of pullbacks.

The stack property for $\mathcal{M}(\mathcal{O})$ follows easily from the stack property for $\mathcal{M}$. The morphism $q$ is defined by forgetting the data of the section $\gamma$ in the obvious way.

Lemma 2.4. The stack $\mathcal{M}(\mathcal{O})$ is algebraic and the morphism $q$ is representable and of finite type.

Proof. Consider a scheme $S$ with a morphism $h: S \rightarrow \mathcal{M}$ corresponding to a family of sheaves $E$ on $S \times M$. Form the fibre product of stacks

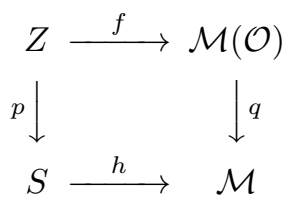

We claim that the stack $Z$ is representable by a scheme of finite type over $S$. This is enough, since if we take $S$ to be an atlas for $\mathcal{M}$, the scheme $Z$ becomes an atlas for $\mathcal{M}(\mathcal{O})$.

It is easy to see that $Z$ is fibered in sets and corresponds to a functor

$$
Z:(\operatorname{Sch} / S)^{\text {op }} \rightarrow \text { Set }
$$

that sends a morphism $f: T \rightarrow S$ to the space of sections

$$
H^{0}\left(T \times M, f^{*}(E)\right) \text {. }
$$

Standard argument allow us to reduce to the case when $S$ is noetherian. Results of Grothendieck [22, Theorem 5.8] then show that this functor is representable by a linear scheme of finite type over $S$.

We shall need the following result about the fibres of the map $q$.

Lemma 2.5. There is a stratification of $\mathcal{M}$ by locally closed substacks

$$
\mathcal{M}_{r} \subset \mathcal{M}
$$

such that the objects of $\mathcal{M}_{r}(\mathbb{C})$ are coherent sheaves $E \in \mathcal{A}$ with

$$
\operatorname{dim}_{\mathbb{C}} H^{0}(M, E)=r .
$$

The pullback of the morphism $q$ to $\mathcal{M}_{r}$ is a locally trivial fibration in the Zariski topology, with fibre $\mathbb{C}^{r}$.

Proof. Given a noetherian scheme $S$ and an $S$-flat coherent sheaf $E$ on $S \times M$, Grothendieck showed [22, Theorem 5.7] that there is a coherent sheaf $G(E)$ on $S$ together with an isomorphism of functors

$$
\mathcal{H o m}_{\mathcal{O}_{S}}(G(E),-) \cong \pi_{S, *}\left(E \otimes_{\mathcal{O}_{S \times M}} \pi_{S}^{*}(-)\right): \operatorname{Coh}(S) \rightarrow \operatorname{Coh}(S) .
$$

The universal property shows that such a sheaf $G(E)$ is unique up to a unique isomorphism and is well-behaved under pullback, in the sense that if $f: T \rightarrow S$ is a morphism of schemes, then there is a natural isomorphism

$$
G\left(f^{*}(E)\right) \cong f^{*}(G(E)) .
$$

It follows from the existence and basic properties of flattening stratifications (see e.g. 22, Theorem 5.13]) that there is a stratification of $S$ by locally closed subschemes $S_{r} \subset S$ on which $G(E)$ is locally free of rank $r$. Moreover these stratifications 
behave well under pullback. It follows that the stack $\mathcal{M}$ has a stratification as claimed.

Using the same notation as in the proof of Lemma 2.4 if the morphism $h$ factors via the inclusion $\mathcal{M}_{r} \subset \mathcal{M}$, the stack $Z$ is represented by the total space of the vector bundle associated to the locally free sheaf $G(E)$. It is therefore a locally trivial fibration with fibre $\mathbb{C}^{r}$.

2.4. Moduli of stable pairs. We shall view the Hilbert scheme and the moduli space of stable pairs as open substacks of the moduli stack $\mathcal{M}(\mathcal{O})$.

Lemma 2.6. There are open substacks

$$
\operatorname{Hilb}_{M} \subset \mathcal{M}(\mathcal{O}), \quad \operatorname{Hilb}_{M}^{\sharp} \subset \mathcal{M}(\mathcal{O})
$$

whose $\mathbb{C}$-valued points are morphisms

$$
\gamma: \mathcal{O}_{M} \rightarrow E
$$

that are epimorphisms in the category $\mathcal{A}$ or $\mathcal{A}^{\sharp}$, respectively.

Proof. We just prove the stable pair case; the Hilbert scheme case is easier and well known. Suppose given a morphism

$$
\gamma: \mathcal{O}_{S \times M} \rightarrow E
$$

as in (4). Given a point $s \in S(\mathbb{C})$, let $\gamma_{s}: \mathcal{O}_{M} \rightarrow E_{s}$ denote the pullback of $\gamma$. We must show that the set of points $s \in S(\mathbb{C})$ for which

(a) the sheaf $E_{s}$ is pure of dimension 1,

(b) the sheaf $\operatorname{coker}\left(\gamma_{s}\right)$ is supported in dimension 0

is the set of $\mathbb{C}$-valued points of an open subscheme of $S$. Standard arguments mean that we can assume $S$ noetherian.

Property (a) is known to be open (see e.g. 10, Prop. 2.3.1]). For property (b), set $Q=\operatorname{coker}(\gamma)$. Then

$$
Q_{s} \cong \operatorname{coker}\left(\gamma_{s}\right)
$$

and so by the basic properties of flattening stratifications (see e.g. 22, Theorem 5.13]) the set of points $s \in S(\mathbb{C})$ for which $\operatorname{coker}\left(\gamma_{s}\right)$ has positive-dimensional support is the set of $\mathbb{C}$-valued points of a closed subscheme of $S$.

The substack Hilb $_{M}$ is represented by the Hilbert scheme. The substack Hilb ${ }_{M}^{\sharp}$ is presumably also represented by a scheme, although we have not checked this. Pandharipande and Thomas [23, Section 1] used results of Le Potier to show that the open and closed substack

$$
\operatorname{Hilb}_{M, \leqslant 1}^{\sharp} \subset \operatorname{Hilb}_{M}^{\sharp}
$$

parameterizing framed sheaves supported in dimension at most 1 is representable.

\section{BEHREND FUNCTION IDENTITY}

Recall [1] that Behrend defined for every scheme $S$ of finite type over $\mathbb{C}$ a constructible function

$$
\nu_{S}: S \rightarrow \mathbb{Z}
$$

with the property that when $f: T \rightarrow S$ is a smooth morphism of relative dimension $d$, there is an identity

$$
\nu_{T}=(-1)^{d} f^{*}\left(\nu_{S}\right)
$$


Using this identity, it is easy [16, Prop. 4.4] to extend Behrend's construction to give a locally constructible function for any Artin stack locally of finite type over $\mathbb{C}$. The identity (6) then also holds for smooth morphisms of stacks.

In this section we establish a Behrend function identity that we need for the proof of Theorem 1.1. The basic idea for the proof came from unpublished notes of Pandharipande and Thomas; nonetheless the author takes full responsibility for the details!

Theorem 3.1. Suppose $\gamma: \mathcal{O}_{M} \rightarrow E$ is a morphism of sheaves such that

(a) $E$ is supported in dimension $\leqslant 1$,

(b) $\operatorname{coker}(\gamma)$ is supported in dimension zero.

Then there is an equality of Behrend functions

$$
\nu_{\mathcal{M}(\mathcal{O})}(\gamma)=(-1)^{\chi(E)} \cdot \nu_{\mathcal{M}}(E) .
$$

Proof. By Lemma 2.5. the morphism $q$ is a vector bundle over the open and closed substack of $\mathcal{M}$ parameterizing zero-dimensional sheaves, so if $E$ is supported in dimension 0, the result follows from (6). Thus we can assume that $E$ has positivedimensional support.

Let $\mathcal{O}_{C} \subset E$ be the image of $\gamma$. It is the structure sheaf of a subscheme $C \subset M$ of dimension 1. Take an ample line bundle $L$ such that

$$
H^{i}(M, E \otimes L)=0 \quad \text { for all } i>0
$$

and a divisor $H \in|L|$ such that $H$ meets $C$ at finitely many pure-dimension 1 points, not in the support of $\operatorname{coker}(\gamma)$.

There is a short exact sequence

$$
0 \longrightarrow \mathcal{O}_{M} \stackrel{s}{\longrightarrow} L \longrightarrow \mathcal{O}_{H}(H) \longrightarrow 0
$$

where $s$ is the section of $L$ corresponding to the divisor $H$. Tensoring it with $E$ and using the above assumptions gives a diagram of sheaves

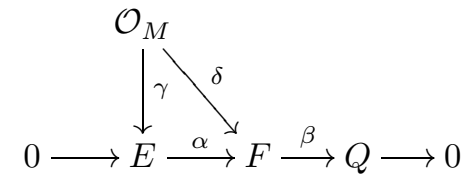

where $F=E \otimes L$. The support of the sheaf $Q$ is zero-dimensional, disjoint from the support of $\operatorname{coker}(\gamma)$, and contained in the pure-dimension 1 part of $C$. In particular,

$$
\operatorname{Hom}_{M}(Q, F)=0 .
$$

Consider the two points of the stack $\mathcal{M}(\mathcal{O})$ corresponding to the maps

$$
\gamma: \mathcal{O}_{M} \rightarrow E, \quad \delta: \mathcal{O}_{M} \rightarrow F .
$$

The statement of the theorem holds for the map $\delta$ because Lemma 2.5 together with (7) implies that

$$
q: \mathcal{M}(\mathcal{O}) \rightarrow \mathcal{M}
$$

is smooth of relative dimension $\chi(F)=H^{0}(M, F)$ over an open neighbourhood of the point $F \in \mathcal{M}(\mathbb{C})$. On the other hand, tensoring sheaves with $L$ defines an automorphism of $\mathcal{M}$, so the Behrend function of $\mathcal{M}$ at the points corresponding to $E$ and $F$ is equal. Thus to prove the theorem, it suffices to show that

$$
(-1)^{\chi(E)} \cdot \nu_{\mathcal{M}(\mathcal{O})}(\gamma)=(-1)^{\chi(F)} \cdot \nu_{\mathcal{M}(\mathcal{O})}(\delta) \text {. }
$$


Consider the stack $X$ whose $S$-valued points are diagrams of $S$-flat sheaves on $S \times M$ of the form

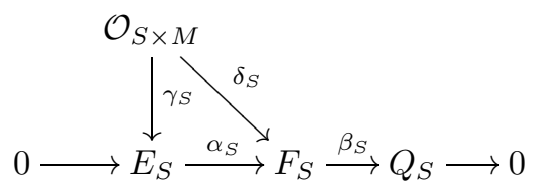

This is an algebraic stack, locally of finite type over $\mathbb{C}$. The easiest way to see this is to write $X$ as a fibre product in a similar way to the stack $Z$ of Lemma 4.3 below. There are two morphisms

$$
p: X \rightarrow \mathcal{M}(\mathcal{O}), \quad q: X \rightarrow \mathcal{M}(\mathcal{O})
$$

taking such a diagram to the maps $\gamma_{S}$ and $\delta_{S}$, respectively. Passing to an open substack of $X$, we can assume that (9) holds at all $\mathbb{C}$-valued points of $X$. It follows easily that $p$ and $q$ induce injective maps on stabilizer groups of $\mathbb{C}$-valued points and hence are representable.

Using (6), it will be enough to show that at the point $x \in X(\mathbb{C})$ corresponding to the diagram (8), the morphisms $p$ and $q$ are smooth of relative dimension $\chi(Q)$ and 0 , respectively. Suppose $f: X \rightarrow Y$ is a representable morphism of stacks, with $X$ and $Y$ locally of finite type over $\mathbb{C}$. Then $f$ is smooth at a point $x \in X(\mathbb{C})$ precisely if the following lifting property is satisfied. Let

$$
i: S \hookrightarrow T
$$

be a closed embedding of affine schemes, corresponding to a homomorphism of Artinian local rings $A \rightarrow A / I$ with $I^{2}=0$. Then given a commutative diagram

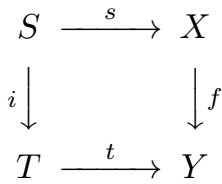

taking the closed point of $S$ to $x$, there is a lifting of $t$ to a morphism $u: T \rightarrow X$ satisfying $f \circ u \cong t$. This follows immediately from the corresponding characterization of smooth morphisms of schemes [8, IV.17.14.2].

Consider the morphism $q$ first. Suppose that we are given a diagram of the form (10), whose pullback to the closed point of $S$ is isomorphic to the diagram (8). Suppose also given a morphism of $T$-flat sheaves

$$
\delta_{T}: \mathcal{O}_{T \times M} \rightarrow F_{T}
$$

on $T \times M$ which when pulled back to $S$ is isomorphic to $\delta_{S}$. Then we must include $\delta_{T}$ in a diagram

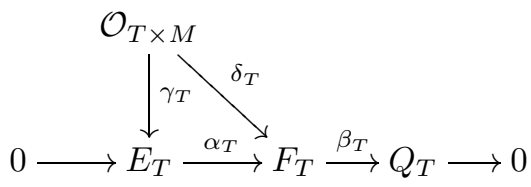

which becomes isomorphic to (10) when pulled back to $S$.

Note that it will be enough to prove the existence of such a lift on an affine open subset $U \subset M$ containing the support of $Q$. Indeed, we can then take another open subset $V \subset M$ disjoint from the support of $Q$ such that $M=U \cup V$. Over the 
subset $V$ the extension problem is trivial and we can glue the results to obtain a lift over $M$.

Let us therefore replace $M$ by $U$. By our assumptions on the support of $Q$, we can then assume that the morphism $\gamma$ is surjective, so that $E \cong \mathcal{O}_{C}$, and that the curve $C$ is of pure-dimension 1 . We can further assume that the line bundle $L$ restricted to $C$ is trivial and hence that we also have $F \cong \mathcal{O}_{C}$.

Let $E_{T}$ be the image of $\delta_{T}$ and fill in the diagram (11). By Lemma 3.2 applied with $S=T$ all the sheaves appearing are flat over $T$. It is then automatic that the diagram becomes isomorphic to (10) when pulled back to $S$, because the morphisms $\beta_{S}$ and $\beta_{T}$ are the cokernels of $\delta_{S}$ and $\delta_{T}$, respectively, and the pullback functor preserves cokernels. The lift we constructed is easily seen to be unique, and hence $q$ is étale.

Let us now check the lifting property for the map $p$. Take a diagram (10) again, and suppose this time that we are given a morphism of $T$-flat sheaves

$$
\gamma_{T}: \mathcal{O}_{T \times M} \rightarrow E_{T}
$$

on $T \times M$ which when pulled back to $S$ is isomorphic to $\gamma_{S}$. We must include $\gamma_{T}$ in a diagram of the form (11) which becomes isomorphic to (10) when pulled back to $S$. As before it is enough perform this lifting over an open affine subset $U \subset M$ containing the support of $Q$.

By the first part of the proof of Lemma 3.2 we can assume that $E_{T} \cong \mathcal{O}_{C_{T}}$ for some closed subscheme $C_{T} \subset T \times U$ which must be flat over $T$. By Lemma 3.2 the sequence in (11) takes the form

$$
0 \longrightarrow \mathcal{O}_{C_{S}} \longrightarrow \mathcal{O}_{C_{S}} \longrightarrow Q_{S} \longrightarrow 0
$$

and thus defines a point of the relative Hilbert scheme of the scheme $C_{S} / S$. This is smooth at the given point because the obstruction space [7, Section 5] is

$$
\operatorname{Ext}_{C}^{1}\left(\mathcal{O}_{C}, Q\right)=0 .
$$

Thus we can extend the sequence over $T$ to give a sequence

$$
0 \longrightarrow K_{T} \longrightarrow \mathcal{O}_{C_{T}} \longrightarrow Q_{T} \longrightarrow 0 .
$$

Applying Lemma 3.2 again with $S=T$ shows that $K_{T} \cong \mathcal{O}_{C_{T}}$ and so we obtain the required extension. In the case that

$$
S=\operatorname{Spec}(\mathbb{C}), \quad T=\operatorname{Spec} \mathbb{C}[\epsilon] /\left(\epsilon^{2}\right),
$$

the set of possible extensions is the tangent space

$$
\operatorname{Hom}_{C}\left(\mathcal{O}_{C}, Q\right)=\mathbb{C}^{\chi(Q)},
$$

and hence $p$ is smooth of this relative dimension.

Lemma 3.2. Suppose $S$ is the spectrum of an Artinian local $\mathbb{C}$-algebra with residue field $\mathbb{C}$, and let $U \subset M$ be an open affine subscheme. Suppose given a short exact sequence of coherent sheaves

$$
0 \longrightarrow E_{S} \longrightarrow F_{S} \longrightarrow Q_{S} \longrightarrow 0
$$

on $S \times U$, with $F_{S}$ flat over $S$, which on restricting to the special fibre gives a sequence of the form

$$
\cdots \longrightarrow \mathcal{O}_{Y} \longrightarrow \mathcal{O}_{Z} \longrightarrow Q \longrightarrow 0
$$


with $Z \subset U$ a Cohen-Macaulay curve and $Q$ supported in dimension 0 . Then all the sheaves appearing in (12) are $S$-flat, and

$$
E_{S} \cong F_{S} \cong \mathcal{O}_{Z_{S}}
$$

for some closed subscheme $Z_{S} \subset S \times U$.

Proof. Since $U$ is affine, we can lift the epimorphism $\mathcal{O}_{U} \rightarrow \mathcal{O}_{Y}$ to a morphism $\mathcal{O}_{S \times U} \rightarrow E_{S}$. This is then surjective by Nakayama's lemma. The same argument applies to $F_{S}$. Thus

$$
E_{S} \cong \mathcal{O}_{Y_{S}}, \quad F_{S} \cong \mathcal{O}_{Z_{S}}
$$

for subschemes $Y_{S}, Z_{S} \subset S \times U$. The inclusion $E_{S} \subset F_{S}$ implies that $Y_{S} \subset Z_{S}$ and hence there is a surjection

$$
\mathcal{O}_{Z_{S}} \rightarrow \mathcal{O}_{Y_{S}}
$$

When restricted to the special fibre, this becomes a surjection $\mathcal{O}_{Z} \rightarrow \mathcal{O}_{Y}$ which is an isomorphism away from the support of $Q$. Since $Q$ has dimension zero and $Z$ is Cohen-Macaulay, this must be an isomorphism. Thus $Z=Y$. Restricting (12) to the special fibre and using purity of $\mathcal{O}_{Y}$ shows that $Q_{S}$ and hence also $E_{S}$ are flat over $S$. If $K_{S}$ is the kernel of the surjection (13), then restricting to the special fibre and using flatness of $E_{S}$ shows that $K_{S}=0$.

\section{Hall Algebra identities}

Our strategy for proving Theorem 1.1 is to first prove identities in a motivic Hall algebra and to then apply an integration map to obtain identities of generating functions for DT invariants. In this section we prove some identities in an infinitetype version of the Hall algebra.

We start by briefly recalling the definition of the motivic Hall algebra $\mathrm{H}(\mathcal{A})$. We refer the reader to [3, Section 3.4] for more details. We then introduce an infinitetype version of the Hall algebra $\mathrm{H}_{\infty}(\mathcal{A})$. This provides a simple context in which to state the identities of this section without having to worry about convergence issues. Although these identities themselves will not be used in the rest of the paper, the arguments used to prove them will appear several times later on.

One difference from [3] which will be important later (see Theorem 6.3) is that we will always take our Grothendieck groups to be defined with complex rather than integral coefficients (we could also have taken rational coefficients).

4.1. Motivic Hall algebra. Suppose $S$ is a stack, locally of finite type over $\mathbb{C}$ and with affine stabilizers. There is a relative Grothendieck group $K(\mathrm{St} / \mathrm{S})$ defined as the complex vector space spanned by equivalence classes of symbols of the form

$$
[X \stackrel{f}{\longrightarrow} S]
$$

with $X$ a stack of finite type over $\mathbb{C}$ with affine stabilizers and $f$ a morphism of stacks, modulo the following relations:

(a) for every pair of stacks $X$ and $Y$ a relation

$$
\left[X_{1} \amalg X_{2} \stackrel{f_{1} \sqcup f_{2}}{\longrightarrow} S\right]=\left[X_{1} \stackrel{f_{1}}{\longrightarrow} S\right]+\left[X_{2} \stackrel{f_{2}}{\longrightarrow} S\right],
$$


(b) for every commutative diagram

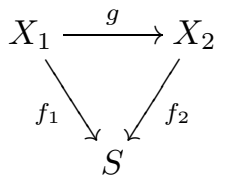

with $g$ an equivalence on $\mathbb{C}$-valued points, a relation

$$
\left[X_{1} \stackrel{f_{1}}{\longrightarrow} S\right]=\left[X_{2} \stackrel{f_{2}}{\longrightarrow} S\right]
$$

(c) for every pair of Zariski locally trivial fibrations

$$
h_{1}: X_{1} \rightarrow Y, \quad h_{2}: X_{2} \rightarrow Y
$$

with the same fibres and every morphism $g: Y \rightarrow S$, a relation

$$
\left[X_{1} \stackrel{g \circ h_{1}}{\longrightarrow} S\right]=\left[X_{2} \stackrel{g \circ h_{2}}{\longrightarrow} S\right] .
$$

The group $K(\mathrm{St} / \mathrm{S})$ has the structure of a $K(\mathrm{St} / \mathbb{C})$-module defined by setting

$$
[X] \cdot[Y \stackrel{f}{\longrightarrow} S]=\left[X \times Y \stackrel{f \circ \pi_{2}}{\longrightarrow} S\right]
$$

and extending linearly.

The motivic Hall algebra [3, Section 4] is the vector space

$$
\mathrm{H}(\mathcal{A})=K(\mathrm{St} / \mathcal{M})
$$

equipped with a noncommutative product given explicitly by the rule

$$
\left[X_{1} \stackrel{f_{1}}{\longrightarrow} \mathcal{M}\right] *\left[X_{2} \stackrel{f_{2}}{\longrightarrow} \mathcal{M}\right]=[Z \stackrel{b \circ h}{\longrightarrow} \mathcal{M}],
$$

where $h$ is defined by the Cartesian square

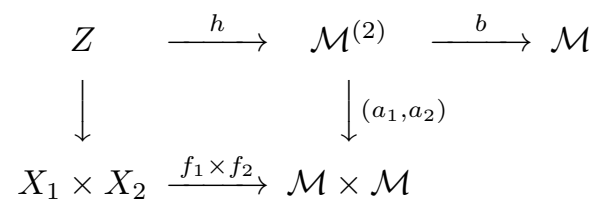

It is an algebra over $K(\mathrm{St} / \mathbb{C})$.

4.2. Infinite-type version. Given a stack $S$ as before, define an infinite-type Grothendieck group $\mathrm{L}\left(\mathrm{St}_{\infty} / \mathrm{S}\right)$ by considering symbols (14) as above, but with $X$ only assumed to be locally of finite type over $\mathbb{C}$. We also drop the relation (a) since otherwise one gets the zero vector space. The infinite-type Hall algebra is then the vector space

$$
\mathrm{H}_{\infty}(\mathcal{A})=\mathrm{L}\left(\mathrm{St}_{\infty} / \mathcal{A}\right)
$$

with the convolution product defined exactly as before.

We shall need some notation for various particular elements of $\mathrm{H}_{\infty}(\mathcal{A})$. Given an open substack $\mathcal{N} \subset \mathcal{M}$, we write

$$
1_{\mathcal{N}}=[\mathcal{N} \stackrel{i}{\longrightarrow} \mathcal{M}] \in \mathrm{H}_{\infty}(\mathcal{A}),
$$

where $i: \mathcal{N} \rightarrow \mathcal{M}$ denotes the inclusion map. Pulling back the morphism (3) to $\mathcal{N} \subset \mathcal{M}$ gives a stack $\mathcal{N}(\mathcal{O})$ with a morphism $q: \mathcal{N}(\mathcal{O}) \rightarrow \mathcal{N}$ and hence an element

$$
1_{\mathcal{N}}^{\mathcal{O}}=[\mathcal{N}(\mathcal{O}) \stackrel{q}{\longrightarrow} \mathcal{M}] \in \mathrm{H}_{\infty}(\mathcal{A}) .
$$


We abuse notation by using the same symbol for an open substack of $\mathcal{M}$ and the corresponding full subcategory of $\mathcal{A}$ defined by its $\mathbb{C}$-valued points. Thus, for example, by [10, Prop. 2.3.1], the objects of the categories $\mathcal{P}$ and $\mathcal{Q}$ of Section 2.2 are the $\mathbb{C}$-valued points of open substacks $\mathcal{P} \subset \mathcal{M}$ and $\mathcal{Q} \subset \mathcal{M}$, and there are corresponding elements

$$
1_{\mathcal{P}}, 1_{\mathcal{Q}} \in \mathrm{H}_{\infty}(\mathcal{A}) .
$$

Finally, restricting $q$ to the open substacks of Lemma2.6 defines further elements

$$
\mathcal{H}=\left[\operatorname{Hilb}_{M} \stackrel{q}{\longrightarrow} \mathcal{M}\right] \in \mathrm{H}_{\infty}(\mathcal{A}), \quad \mathcal{H}^{\sharp}=\left[\operatorname{Hilb}_{M}^{\sharp} \stackrel{q}{\longrightarrow} \mathcal{M}\right] \in \mathrm{H}_{\infty}(\mathcal{A}) .
$$

We will now establish some identities in the ring $\mathrm{H}_{\infty}(\mathcal{A})$ relating these elements.

\subsection{Torsion pair identities.}

Lemma 4.1. There is an identity

$$
1_{\mathcal{M}}=1_{\mathcal{P}} * 1_{\mathcal{Q}} .
$$

Proof. Form a Cartesian square

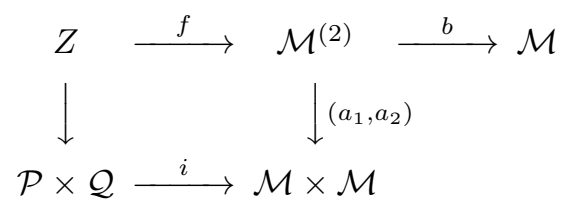

where the map $i$ is the open embedding. Since the morphism $\left(a_{1}, a_{2}\right)$ satisfies the iso-fibration property of [3, Lemma A.1], the groupoid of $S$-valued points of $Z$ can be described as follows. The objects are short exact sequences of $S$-flat sheaves on $S \times M$ of the form

$$
0 \longrightarrow P \longrightarrow E \longrightarrow Q \longrightarrow 0
$$

such that $P$ and $Q$ define flat families of sheaves on $M$ lying in the subcategories $\mathcal{P}$ and $\mathcal{Q}$, respectively. The morphisms are isomorphisms of short exact sequences. The composition

$$
g=b \circ f: Z \rightarrow \mathcal{M}
$$

sends such a short exact sequence to the object $E$. This morphism induces an equivalence on $\mathbb{C}$-valued points because of the torsion pair property: every object $E$ of $\mathcal{A}$ fits into a unique short exact sequence of the form (16). Thus the identity follows from relation (b) above.

Lemma 4.2. There is an identity

$$
1_{\mathcal{M}}^{\mathcal{O}}=1_{\mathcal{P}}^{\mathcal{O}} * 1_{\mathcal{Q}}^{\mathcal{O}}
$$

Proof. Form Cartesian squares

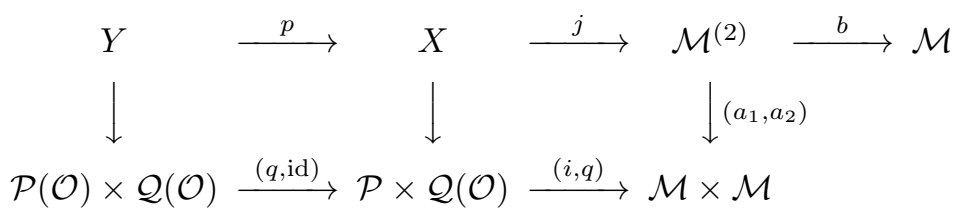

Then $1_{\mathcal{P}}^{\mathcal{O}} * 1_{Q}^{\mathcal{O}}$ is represented by the composite map $b \circ j \circ p: Y \rightarrow \mathcal{M}$. Note that, by Lemma 2.5, the map

$$
q: \mathcal{P}(\mathcal{O}) \rightarrow \mathcal{P}
$$


is a Zariski locally trivial fibration, with fibre over a zero-dimensional sheaf $P$ being the vector space $H^{0}(M, P)$. By pullback the same is true of the morphism $p$.

The groupoid of $S$-valued points of $X$ is as follows. The objects are short exact sequences of $S$-flat sheaves on $S \times M$ of the form

$$
0 \longrightarrow P \stackrel{\alpha}{\longrightarrow} E \stackrel{\beta}{\longrightarrow} Q \longrightarrow 0
$$

such that $P$ and $Q$ define flat families of sheaves on $M$ lying in the subcategories $\mathcal{P}$ and $\mathcal{Q}$, respectively, together with a map

$$
\gamma: \mathcal{O}_{S \times M} \rightarrow Q
$$

The morphisms are isomorphisms of short exact sequences commuting with the map $\gamma$. We can represent these objects as diagrams

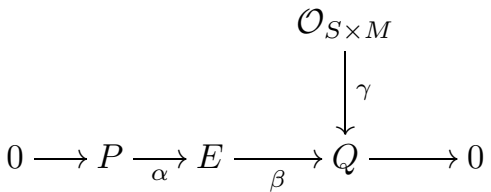

Consider the stack $Z$ of Lemma 4.1 with its map $g: Z \rightarrow \mathcal{M}$ and form a Cartesian square

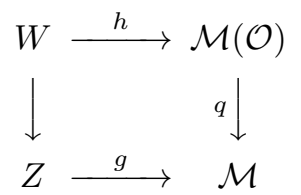

Since $g$ induces an equivalence on $\mathbb{C}$-valued points, so too does $h$, so the element $1_{\mathcal{M}}^{\mathcal{O}}$ can be represented by the morphism $q \circ h$.

The groupoid of $S$-valued points of $W$ can be represented by diagrams

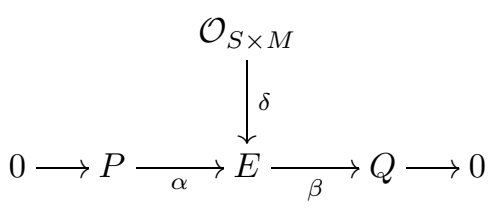

Setting $\gamma=\beta \circ \delta$ defines a morphism of stacks $W \rightarrow X$. It is easy to see that this is a Zariski locally trivial fibration, with fibre over a $\mathbb{C}$-valued point of $X$ represented by a diagram (17) being an affine space for $H^{0}(M, P)$. Since this is the same fibre as the map $p$, the result follows from relation (c) above.

4.4. Hilbert scheme identity. The next result is analogous to an identity proved by Engel and Reineke [5, Lemma 5.1] in the context of Hall algebras of quiver representations defined over finite fields.

Lemma 4.3. There is an identity

$$
1_{\mathcal{M}}^{\mathcal{O}}=\mathcal{H} * 1_{\mathcal{M}} .
$$

Proof. Form a Cartesian square

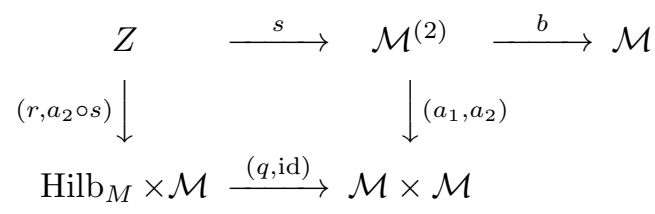


The groupoid of $S$-valued points of $Z$ can be described as follows. The objects are short exact sequences of $S$-flat sheaves on $S \times M$

$$
0 \longrightarrow A \stackrel{\alpha}{\longrightarrow} E \stackrel{\beta}{\longrightarrow} B \longrightarrow 0
$$

together with an epimorphism $\gamma: \mathcal{O}_{S \times M} \rightarrow A$. The morphisms are isomorphisms of such short exact sequences commuting with the maps from $\mathcal{O}_{S \times M}$. We can represent these objects as diagrams of the form

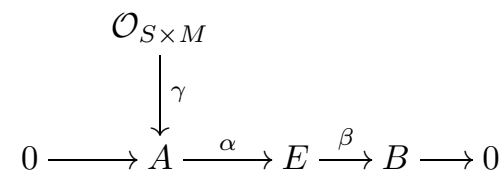

There is a morphism of stacks $h: Z \rightarrow \mathcal{M}(\mathcal{O})$ sending a diagram (19) to the composite map

$$
\delta=\alpha \circ \gamma: \mathcal{O}_{S \times M} \rightarrow E .
$$

This morphism fits into a commuting diagram of stacks

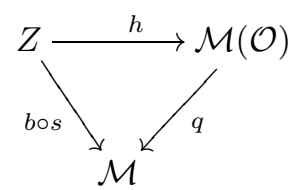

Then $h$ induces an equivalence on $\mathbb{C}$-valued points, because every map $\delta: \mathcal{O}_{M} \rightarrow E$ in $\mathcal{A}$ factors uniquely as an epimorphism $\gamma: \mathcal{O}_{M} \rightarrow A$ followed by a monomorphism $\alpha: A \hookrightarrow E$.

\subsection{Stable pair identity.}

Lemma 4.4. There is an identity

$$
1_{\mathcal{Q}}^{\mathcal{O}}=\mathcal{H}^{\sharp} * 1_{\mathcal{Q}} .
$$

Proof. This is similar to Lemma 4.3. We consider the Cartesian square

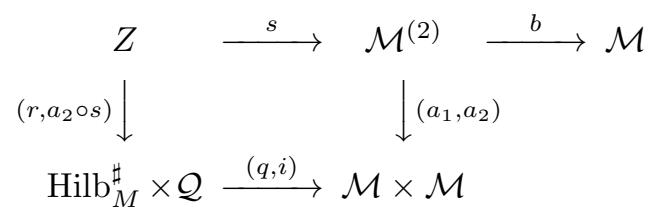

This time the objects in the groupoid of $S$-valued points of $W$ are diagrams of $S$-flat sheaves on $S \times M$ of the form

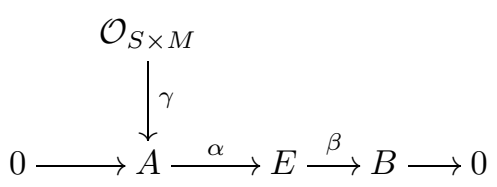

such that for each point $s \in S(\mathbb{C})$, the pullback $B_{s}$ is in $\mathcal{Q}$ and the map $\gamma_{s}: \mathcal{O}_{M} \rightarrow$ $A_{s}$ is an epimorphism in $\mathcal{A}^{\sharp}$. In particular, by Lemma 2.3 this implies that $A_{s}$ is in $\mathcal{Q}$ and hence so too is $E_{s}$. 
There is a morphism $h: Z \rightarrow \mathcal{Q}(\mathcal{O})$ sending such an object to the composite morphism $\delta=\alpha \circ \gamma$ as before and a commutative diagram

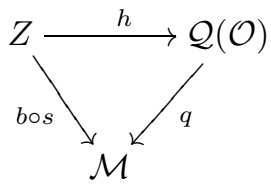

Once again $h$ is an equivalence on $\mathbb{C}$-valued points, this time because if $E \in \mathcal{Q}$, then every map $\mathcal{O}_{M} \rightarrow E$ factors uniquely as an epimorphism $\mathcal{O}_{M} \rightarrow A$ in $\mathcal{A}^{\sharp}$ followed by a monomorphism $A \rightarrow E$ in $\mathcal{A}^{\sharp}$. By Lemma 2.3, one then has $A \in \mathcal{Q}$, and forming the short exact sequence

$$
0 \longrightarrow A \longrightarrow E \longrightarrow B \longrightarrow 0
$$

in $\mathcal{A}^{\sharp}$ and applying the argument of Lemma 2.3 gives $B \in \mathcal{Q}$ also.

\section{Hall algebras of Dimension one Sheaves}

In this section we assemble some general properties of Hall algebras of coherent sheaves on $M$ supported in dimension $\leqslant 1$. We first adapt some material from $[\underline{3}$ to this context and then introduce a formal completion operation which allows us to treat the infinite sums of invariants appearing in Theorem 1.1. Finally, we consider the properties of the dualizing functor applied to pure-dimension 1 sheaves.

\subsection{Hall algebra and integration map. We write}

$$
\mathcal{C}=\operatorname{Coh}_{\leqslant 1}(M) \subset \operatorname{Coh}(M)=\mathcal{A}
$$

for the full subcategory consisting of sheaves with support of dimension $\leqslant 1$. By our usual abuse of notation, this corresponds to an open and closed substack

$$
\mathcal{C} \subset \mathcal{M} \text {. }
$$

As in [3, Section 4.4] there is a $K(\mathrm{St} / \mathcal{C})$-subalgebra $\mathrm{H}(\mathcal{C}) \subset \mathrm{H}(\mathcal{A})$ spanned by symbols

$$
[X \stackrel{f}{\longrightarrow} \mathcal{M}]
$$

factoring via the inclusion $\mathcal{C} \subset \mathcal{M}$. As explained in [3, Section 5.1] there is a $K(\operatorname{Var} / \mathbb{C})\left[\mathbb{L}^{-1}\right]$-subalgebra

$$
\mathrm{H}_{\text {reg }}(\mathcal{C}) \subset \mathrm{H}(\mathcal{C})
$$

spanned by symbols (23) with $X$ a scheme. Elements of this subalgebra are called regular. The quotient algebra

$$
\mathrm{H}_{\mathrm{sc}}(\mathcal{C})=\mathrm{H}_{\text {reg }}(\mathcal{C}) /(\mathbb{L}-1) \mathrm{H}_{\text {reg }}(\mathcal{C})
$$

is commutative and is equipped with a Poisson bracket

$$
\{f, g\}=\frac{f * g-g * f}{\mathbb{L}-1} \bmod (\mathbb{L}-1) .
$$

All these algebras are graded by the commutative monoid $\Delta$, the basic reason being the decomposition

$$
\mathcal{C}=\coprod_{\alpha \in \Delta} \mathcal{C}_{\alpha}
$$

into open and closed substacks paramaterizing sheaves with a fixed Chern character. 
Let $\mathbb{C}[\Delta]$ denote the monoid algebra of $\Delta$ over $\mathbb{C}$. It has basis given by symbols $x^{\alpha}$ for $\alpha \in \Delta$ with product defined by

$$
x^{\alpha} * x^{\beta}=x^{\alpha+\beta} .
$$

Since the Euler form on $N_{\leqslant 1}(M)$ is trivial, we equip $\mathbb{C}[\Delta]$ with the trivial Poisson bracket.

Theorem 5.1. There is a homomorphism of $\Delta$-graded Poisson algebras

$$
I: \mathrm{H}_{\mathrm{sc}}(\mathcal{C}) \rightarrow \mathbb{C}[\Delta]
$$

with the property that

$$
I\left(\left[X \stackrel{f}{\longrightarrow} \mathcal{C}_{\alpha}\right]\right)=\chi\left(X, f^{*}\left(\nu_{\mathcal{C}}\right)\right) \cdot x^{\alpha},
$$

where $\nu_{\mathcal{C}}: \mathcal{C} \rightarrow \mathbb{Z}$ is the Behrend function for the stack $\mathcal{C}$.

Proof. This follows immediately from [3, Theorem 5.2] upon restricting to the subalgebra $\mathrm{H}(\mathcal{C}) \subset \mathrm{H}(\mathcal{A})$.

5.2. Laurent subsets. Theorem 1.1 involves Laurent series rather than polynomials. To fit these into our framework, we need to enlarge the algebras $\mathrm{H}(\mathcal{C})$ and $\mathbb{C}[\Delta]$ in a formal way using the grading. In this section we explain how to do this.

Definition 5.2. A subset $S \subset \Delta$ will be called Laurent if for each $\beta \in N_{1}(M)$ the set of integers $n$ for which $(\beta, n) \in S$ is bounded below.

Let $\Phi$ denote the set of Laurent subsets of $\Delta$. By Lemma 2.1 this system of subsets satisfies the following:

(a) If $S, T \in \Phi$, then so is $S+T=\{\alpha+\beta: \alpha \in S, \beta \in T\}$.

(b) If $S, T \in \Phi$ and $\alpha \in \Delta$, there are only finitely many decompositions $\alpha=$ $\beta+\gamma$ with $\beta \in S$ and $\gamma \in T$.

Suppose that $A=\bigoplus_{\gamma \in \Delta} A_{\gamma}$ is a $\Delta$-graded ring. We can form an abelian group $A_{\Phi}$ whose elements are formal sums

$$
a=\sum_{\gamma \in S \subset \Delta} a_{\gamma}
$$

for Laurent subsets $S \in \Phi$. Given an element $a \in A_{\Phi}$ as in (26), we set $\pi_{\gamma}(a)=$ $a_{\gamma} \in A_{\gamma}$. The ring structure on $A$ induces one on $A_{\Phi}$ via the rule

$$
\pi_{\gamma}(a * b)=\sum_{\alpha+\beta=\gamma} \pi_{\alpha}(a) * \pi_{\beta}(b) .
$$

We can equip $A_{\Phi}$ with a topology by stipulating that a sequence $\left(a_{j}\right) \subset A_{\Phi}$ is convergent if for any $(\beta, m) \in \Delta$ there is an integer $K$ such that

$$
i, j \geqslant K \Longrightarrow \pi_{(\beta, n)}\left(a_{i}\right)=\pi_{(\beta, n)}\left(a_{j}\right) \text { for all } n \leqslant m .
$$

It is easy to check using Lemma 2.1 that with this topology $A_{\Phi}$ becomes a topological algebra.

Lemma 5.3. If $A$ is a $\Delta$-graded $\mathbb{C}$-algebra and $a \in A_{\Phi}$ satisfies $\pi_{0}(a)=0$, then any series

$$
\sum_{j \geqslant 1} c_{j} a^{j}
$$

with coefficients $c_{j} \in \mathbb{C}$ is convergent in the topological ring $A_{\Phi}$. In particular the element $1-a$ is invertible. 
Proof. Define a subset

$$
\Delta(a)=\left\{\gamma \in \Delta: \pi_{\gamma}(a) \neq 0\right\} .
$$

Then $\Delta(a)$ is a Laurent subset consisting of nonzero elements. Fix $(\beta, m) \in \Delta$ and consider elements $\gamma=(\beta, n) \in \Delta$ with $n \leqslant m$. It follows from Lemma 2.1 that there are only finitely many decompositions of such an element $\gamma$ into a sum of the form

$$
\gamma=\gamma_{1}+\cdots+\gamma_{k}, \quad \gamma_{i} \in \Delta(a) .
$$

The convergence of the given series follows immediately from this. The final statement follows by considering the geometric series in the usual way.

If $A$ and $B$ are two $\Delta$-graded algebras, then a $\Delta$-graded homomorphism $f: A \rightarrow$ $B$ induces a continuous homomorphism $f_{\Phi}: A_{\Phi} \rightarrow B_{\Phi}$ by setting

$$
\pi_{\gamma}\left(f_{\Phi}(a)\right)=f\left(\pi_{\gamma}(a)\right) .
$$

Note that if $f$ is injective, then so is $f_{\Phi}$. Thus if $A$ is a subring of $B$, we can identify $A_{\Phi}$ with a subring of $B_{\Phi}$.

5.3. $\Phi$-finite elements. Applying the construction of the last section to the Hall algebra gives a $K(\mathrm{St} / \mathbb{C})$-algebra $\mathrm{H}(\mathcal{C})_{\Phi}$ with a $K(\operatorname{Var} / \mathbb{C})\left[\mathbb{L}^{-1}\right]$-subalgebra

$$
\mathrm{H}_{\mathrm{reg}}(\mathcal{C})_{\Phi} \subset \mathrm{H}(\mathcal{C})_{\Phi}
$$

whose elements we again call regular. There is also a commutative quotient algebra

$$
\mathrm{H}_{\mathrm{sc}}(\mathcal{C})_{\Phi}=\mathrm{H}_{\mathrm{reg}}(\mathcal{C})_{\Phi} /(\mathbb{L}-1) \mathrm{H}_{\mathrm{reg}}(\mathcal{C})_{\Phi}
$$

equipped with a Poisson bracket and a homomorphism of Poisson algebras

$$
I_{\Phi}: \mathrm{H}_{\mathrm{sc}}(\mathcal{C})_{\Phi} \rightarrow \mathbb{C}[\Delta]_{\Phi} .
$$

Recall the decomposition (24) of the stack $\mathcal{C}$.

Definition 5.4. A morphism of stacks $f: X \rightarrow \mathcal{C}$ will be called $\Phi$-finite if

(a) $X_{\alpha}=f^{-1}\left(\mathcal{C}_{\alpha}\right)$ is of finite type for all $\alpha \in \Delta$,

(b) there is a subset $S \in \Phi$ such that $X_{\alpha}$ is empty unless $\alpha \in S$.

Any such morphism defines an element of the ring $\mathrm{H}(\mathcal{C})_{\Phi}$ defined by the formal sum

$$
\sum_{\alpha \in S}\left[X_{\alpha} \stackrel{f}{\longrightarrow} \mathcal{C}\right] .
$$

To give some examples, introduce the open and closed subschemes

$$
\operatorname{Hilb}_{M, \leqslant 1}=\mathcal{C} \cap \operatorname{Hilb}_{M}, \quad \operatorname{Hilb}_{M, \leqslant 1}^{\sharp}=\mathcal{C} \cap \operatorname{Hilb}_{M}^{\sharp}
$$

parameterizing quotients of $\mathcal{O}_{M}$ supported in dimension $\leqslant 1$.

Lemma 5.5. The morphisms

$$
q: \operatorname{Hilb}_{M, \leqslant 1} \rightarrow \mathcal{C}, \quad q: \operatorname{Hilb}_{M, \leqslant 1}^{\sharp} \rightarrow \mathcal{C}
$$

are $\Phi$-finite. The corresponding elements $\mathcal{H}_{\leqslant 1}$ and $\mathcal{H}_{\leqslant 1}^{\sharp}$ of $\mathrm{H}(\mathcal{C})_{\Phi}$ satisfy

$$
I_{\Phi}\left(\mathcal{H}_{\leqslant 1}\right)=\sum_{(\beta, n) \in \Delta}(-1)^{n} \mathrm{DT}(\beta, n) x^{\beta} q^{n}=\mathrm{DT}_{\beta}(-q) \cdot x^{\beta}
$$


where we have written $x^{\beta}=x^{(\beta, 0)}$ and $q=x^{(0,1)}$. Similarly

$$
I_{\Phi}\left(\mathcal{H}_{\leqslant 1}^{\sharp}\right)=\sum_{(\beta, n) \in \Delta}(-1)^{n} \mathrm{PT}(\beta, n) x^{\beta} q^{n}=\operatorname{PT}_{\beta}(-q) \cdot x^{\beta} .
$$

Proof. The Hilbert scheme parameterizing quotients of $\mathcal{O}_{M}$ of fixed class $\gamma \in N(M)$ is always of finite type. On the other hand the set of elements $\gamma \in \Delta$ for which $\operatorname{Hilb}_{M}(\gamma)$ is nonempty is Laurent, the basic reason being that the curves lying in a fixed class $\beta \in N_{1}(M)$ have bounded genus. The same argument applies to the stable pair moduli space. The formulae then follow from Theorem 3.1 and Behrend's description of DT invariants as a weighted Euler characteristic.

5.4. Duality functor. There is a full subcategory $\mathcal{Q} \cap \mathcal{C} \subset \mathcal{C}$ consisting of puredimension 1 sheaves.

Lemma 5.6. The functor

$$
\mathbb{D}=\mathcal{E} x t_{\mathcal{O}_{M}}^{2}\left(-, \mathcal{O}_{M}\right): \operatorname{Coh}(M) \rightarrow \operatorname{Coh}(M)
$$

restricts to a contravariant equivalence

$$
\mathbb{D}: \mathcal{Q} \cap \mathcal{C} \rightarrow \mathcal{Q} \cap \mathcal{C}
$$

satisfying $\mathbb{D}^{2} \cong \mathrm{id}$.

Proof. It is immediate from the definition that the functor

$$
\mathbb{D}=\mathbf{R H o m}_{\mathcal{O}_{M}}\left(-, \mathcal{O}_{M}\right)[2]: D^{b} \operatorname{Coh}(M) \rightarrow D^{b} \operatorname{Coh}(M)
$$

is a contravariant equivalence and satisfies $\mathbb{D}^{2} \cong \mathrm{id}$. Thus we must just show that with the given shift $\mathbb{D}$ takes the subcategory $\mathcal{Q} \cap \mathcal{C}$ into itself.

By Serre duality, for any object $E \in \mathcal{Q} \cap \mathcal{C}$ and any point $x \in M$

$$
\operatorname{Ext}_{M}^{3}\left(E, \mathcal{O}_{x}\right)=\operatorname{Hom}_{M}\left(\mathcal{O}_{x}, E\right)^{*}=0,
$$

and so by the usual argument (see e.g. [4, Prop. 5.4]) $E$ has a locally free resolution of length 2. Applying $\mathbb{D}$ gives another length 2 complex of locally free sheaves $\mathbb{D}(E)$ with the same support as $E$. By a standard argument (see e.g. [4, Lemma 4.2]) it follows that $\mathbb{D}(E)$ is concentrated in degree 0 , and (30) then shows that $\mathbb{D}(E)$ is pure.

The functor $\mathbb{D}$ preserves families and hence induces an involution $\mathbb{D}$ of the open substack $\mathcal{Q} \cap \mathcal{C} \subset \mathcal{C}$. There is a $K(\mathrm{St} / \mathbb{C})$-submodule

$$
K(\mathrm{St} / \mathcal{Q} \cap \mathcal{C}) \subset K(\mathrm{St} / \mathcal{C})
$$

spanned by maps (14) factoring via the open substack $\mathcal{Q} \cap \mathcal{C}$. The involution $\mathbb{D}$ defines a $K(\mathrm{St} / \mathbb{C})$-linear involution

$$
\mathbb{D}_{*}: K(\mathrm{St} / \mathcal{Q} \cap \mathcal{C}) \rightarrow K(\mathrm{St} / \mathcal{Q} \cap \mathcal{C}) .
$$

This will ultimately be responsible for the invariance of the DT generating function under the transformation $q \leftrightarrow q^{-1}$.

Lemma 5.7. The submodule $K(\mathrm{St} / \mathcal{Q} \cap \mathcal{C})$ is closed under the convolution product. It therefore defines a subalgebra $\mathrm{H}(\mathcal{Q} \cap \mathcal{C}) \subset \mathrm{H}(\mathcal{C})$. Moreover, one has

$$
\mathbb{D}_{*}(a * b)=\mathbb{D}_{*}(b) * \mathbb{D}_{*}(a),
$$

for any $a, b \in \mathrm{H}(\mathcal{Q} \cap \mathcal{C})$, or in other words, $\mathbb{D}_{*}$ is an anti-involution of $\mathrm{H}(\mathcal{Q} \cap \mathcal{C})$. 
Proof. The first statement is just the fact that the subcategory $\mathcal{Q} \cap \mathcal{C}$ is closed under extensions. The second statement holds because $\mathbb{D}$ is an exact anti-equivalence, which means that if

$$
0 \longrightarrow A_{1} \longrightarrow B \longrightarrow A_{2} \longrightarrow 0
$$

is a short exact sequence in $\mathcal{Q} \cap \mathcal{C}$, then so is

$$
0 \longrightarrow \mathbb{D}\left(A_{2}\right) \longrightarrow \mathbb{D}(B) \longrightarrow \mathbb{D}\left(A_{1}\right) \longrightarrow 0 .
$$

Thus $\mathbb{D}$ induces an automorphism of the stack of short exact sequences in $\mathcal{Q} \cap \mathcal{C}$ permuting the two projections $a_{1}, a_{2}$ to $\mathcal{Q} \cap \mathcal{C}$.

\section{DT/PT CORRESPONDENCE}

In this section we introduce a stability condition on the category $\mathcal{C}$ and state a deep result of Joyce which shows that certain elements relating to characteristic functions of semistable objects are regular. We also prove a Hall algebra identity arising from the existence and uniqueness of Harder-Narasimhan filtrations. These results are then used to prove the first part of Theorem 1.1, the correspondence between Donalson-Thomas and stable pair invariants.

6.1. A stability condition. Choose an ample divisor $H$ on $M$. Given a class $\gamma \in \Delta$ define the slope

$$
\mu(\gamma)=\frac{\operatorname{ch}_{3}(\gamma)}{\operatorname{ch}_{2}(\gamma) \cdot H} \in(-\infty,+\infty] .
$$

If $\operatorname{ch}_{2}(\gamma)=0$, we consider $\gamma$ to have slope $+\infty$, and otherwise $\mu(\gamma) \in \mathbb{Q}$. A nonzero object $E \in \mathcal{C}$ is (Gieseker or Simpson) semistable if

$$
\mu(A) \leqslant \mu(E)
$$

for all nonzero subobjects $A \subset E$. We write $\operatorname{SS}(\gamma) \subset \mathcal{C}$ for the open stack whose $\mathbb{C}$-valued points are semistable sheaves of class $\gamma \in \Delta$.

Every nonzero sheaf $E \in \mathcal{C}$ has a unique Harder-Narasimhan filtration

$$
0=E_{0} \subset E_{1} \subset \cdots \subset E_{n-1} \subset E_{n}=E
$$

whose factors $F_{i}=E_{i} / E_{i-1}$ are semistable with descending slope

$$
\mu\left(F_{1}\right)>\mu\left(F_{2}\right)>\cdots>\mu\left(F_{n}\right) .
$$

Given an interval $I \subset(-\infty,+\infty]$, define $\operatorname{SS}(I) \subset \mathcal{C}$ to be the full subcategory consisting of zero objects together with those sheaves whose Harder-Narasimhan factors all have slope in $I$. Note that there are identifications

$$
\mathcal{P}=\mathrm{SS}(\infty), \quad \mathcal{Q} \cap \mathcal{C}=\operatorname{SS}(-\infty,+\infty) .
$$

By the argument of [10, Prop. 2.3.1], bounding slopes of Harder-Narasimhan factors is an open condition, so for each interval $I$ there is an open substack $\operatorname{SS}(I) \subset$ $\mathcal{M}$. If $I$ is bounded below, then by [10, Theorem 3.3.7] the inclusion functor is $\Phi$ finite and so defines an element

$$
1_{\mathrm{SS}(I)} \in \mathrm{H}(\mathcal{C})_{\Phi} .
$$

In particular, for each $\mu \in(-\infty,+\infty]$ there is a full subcategory $\operatorname{SS}(\mu) \subset \mathcal{C}$ consisting of the zero objects and the semistable objects of slope $\mu$. Since the identity 
in the Hall algebra is represented by the substack $\left[\mathcal{M}_{0} \subset \mathcal{M}\right]$ of zero objects, one has

$$
1_{\mathrm{SS}(\mu)}=1+\sum_{\substack{0 \neq \gamma \in \Delta \\ \mu(\gamma)=\mu}} 1_{\mathrm{SS}(\gamma)} .
$$

It is easy to see that for any sheaf $E \in \mathcal{Q} \cap \mathcal{C}$

$$
\operatorname{ch}(\mathbb{D}(E))=\left(\operatorname{ch}_{2}(E),-\operatorname{ch}_{3}(E)\right) \in \Delta,
$$

which of course implies that

$$
\mu(\mathbb{D}(E))=-\mu(E) .
$$

The following is an easy consequence of this.

Lemma 6.1. Let $I \subset \mathbf{R}$ be a bounded interval. Then

$$
\mathbb{D}(\mathrm{SS}(I))=\mathrm{SS}(-I) \text {. }
$$

Proof. Suppose $E \in \mathcal{Q} \cap \mathcal{C}$. Then $E$ lies in the subcategory $E \in \mathrm{SS}([a, b])$ precisely if for every short exact sequence of objects of $\mathcal{Q} \cap \mathcal{C}$

$$
0 \longrightarrow A \longrightarrow E \longrightarrow B \longrightarrow 0
$$

one has $\mu(A) \leqslant b$ and $\mu(B) \geqslant a$. The result therefore follows from (33).

6.2. Harder-Narasimhan identity. The next result is analogous to an identity proved by Reineke [24, Prop. 4.8] in the context of Hall algebras of quiver representations defined over finite fields. Similar identities play a fundamental role in the study of wall-crossing behaviour in [15, 18.

Lemma 6.2. Assume the interval $I \subset(-\infty,+\infty]$ is bounded below. Then there is an identity

$$
1_{\mathrm{SS}(I)}=\prod_{\mu \in I} 1_{\mathrm{SS}(\mu)} \in \mathrm{H}(\mathcal{C})_{\Phi}
$$

where the infinite product is taken in descending order of slope.

Proof. We first explain what the identity means. Given a finite subset $V \subset I$, we can form a product

$$
1_{\mathrm{SS}(V)}=\prod_{\mu \in V} 1_{\mathrm{SS}(\mu)} \in \mathrm{H}(\mathcal{C})_{\Phi}
$$

where we take the terms in descending order of slope. Suppose that

$$
V_{1} \subset V_{2} \subset \cdots \subset V_{j} \subset \cdots \subset I
$$

is an increasing sequence of subsets whose union contains all rational points of $I$. Then the claim is that the elements $1_{\mathrm{SS}\left(V_{j}\right)}$ converge to $1_{\mathrm{SS}(I)}$.

Fix $(\beta, m) \in \Delta$ and consider elements $\gamma=(\beta, n)$ with $n<m$. By the boundedness assumption on the interval $I$ and Lemma 2.1, there are only finitely many ways of writing such an element as a sum

$$
\gamma=\gamma_{1}+\cdots \gamma_{k}
$$

with each $\gamma_{i} \in \Delta$ and satisfying $\mu\left(\gamma_{i}\right) \in I$. 
The existence and uniqueness of the Harder-Narasimhan filtration together with the formula for repeated products in the Hall algebra (see [3, Lemma 4.2]) implies that there is an identity

$$
\pi_{\gamma}\left(1_{\mathrm{SS}(I)}\right)=\sum_{k \geqslant 1} \sum_{\substack{\gamma_{1}+\cdots+\gamma_{k}=\gamma \\ \mu\left(\gamma_{1}\right)>\ldots, \mu\left(\gamma_{k}\right) \\ \mu\left(\gamma_{i}\right) \in I}} 1_{\mathrm{SS}\left(\gamma_{1}\right)} * \cdots * 1_{\mathrm{SS}\left(\gamma_{k}\right)},
$$

where the sum on the right is finite. Indeed, each term on the right is represented by a stack whose $\mathbb{C}$-valued points paramaterize sheaves $E$ with a Harder-Narasimhan filtration of the given type. By (31) it follows that

$$
\pi_{\gamma}\left(1_{\mathrm{SS}(I)}\right)=\pi_{\gamma}\left(\prod_{\mu \in I} 1_{\mathrm{SS}(\mu)}\right) .
$$

Thus if $j$ is large enough that $V_{j}$ contains the slopes of all $\gamma_{i}$ appearing in decompositions of the form (35), then

$$
\pi_{\gamma}\left(1_{\mathrm{SS}(I)}\right)=\pi_{\gamma}\left(1_{\mathrm{SS}\left(V_{j}\right)}\right)
$$

which proves the claim.

6.3. The no-pole theorem. The following statement is a consequence of deep results of Joyce. It is an analogue of the no-poles conjecture in [18. At this point it becomes important that we have used complex (or at least rational) coefficients to define our Grothendieck rings (rather than the integral coefficients of [3]).

Theorem 6.3. For each $\mu \in(-\infty,+\infty]$ we can write

$$
1_{\mathrm{SS}(\mu)}=\exp \left(\epsilon_{\mu}\right) \in \mathrm{H}(\mathcal{C})_{\Phi}
$$

with $\eta_{\mu}=\left[\mathbb{C}^{*}\right] \cdot \epsilon_{\mu} \in \mathrm{H}_{\mathrm{reg}}(\mathcal{C})_{\Phi}$ a regular element.

Proof. Given $\gamma \in \Delta$, consider the finite sum

$$
\epsilon_{\gamma}=\sum_{k \geqslant 1} \sum_{\substack{\gamma=\gamma_{1}+\cdots+\gamma_{k} \\ \mu\left(\gamma_{i}\right)=\mu(\gamma)}} \frac{(-1)^{k-1}}{k} 1_{\mathrm{SS}\left(\gamma_{1}\right)} * \cdots * 1_{\mathrm{SS}\left(\gamma_{k}\right)} .
$$

Joyce proved that $\left[\mathbb{C}^{*}\right] \cdot \epsilon_{\gamma} \in \mathrm{H}_{\text {reg }}(\mathcal{C})$. By (31) this can be rephrased as the statement that

$$
\left[\mathbb{C}^{*}\right] \cdot \log \left(1_{\mathrm{SS}(\mu)}\right) \in \mathrm{H}_{\mathrm{reg}}(\mathcal{C})_{\Phi},
$$

which is equivalent to the claim made in the statement of the theorem via the inverse properties of the expansions of log and exp.

More precisely [14, Theorem 8.7] shows that $\epsilon_{\gamma} \in \Pi_{1} \mathrm{SF}(\mathcal{M})$, where $\Pi_{1}$ is Joyce's projection onto virtual indecomposables. But using [13, Cor. 5.10] and the definition of the operator $\Pi_{1}$, this means that in the stack function algebra $\overline{\mathrm{SF}}(\mathcal{M}, \Upsilon, \Lambda)$ the element $\epsilon_{\gamma}$ is represented by a sum of elements of the form

$$
\left[X_{i} / \mathbb{C}^{*} \stackrel{f_{i}}{\longrightarrow} \mathcal{M}\right]
$$

with each $X_{i}$ a variety. Taking the motivic invariant to be the canonical map

$$
\Upsilon: K(\operatorname{Var} / \mathbb{C}) \rightarrow K(\mathrm{St} / \mathbb{C})
$$

and using [3, Remark 3.11] show that $\left[\mathbb{C}^{*}\right] \cdot \epsilon_{\gamma}$ is regular as claimed. 
Joyce's proof of this crucial result uses the full force of the technology developed in the long series of papers [11, 12, 13, including moduli spaces of configurations and the virtual projection operators $\Pi_{k}$. In the author's opinion it would be a worthwhile project to try to write down a more conceptual and easily understood proof.

Corollary 6.4. For any $\mu \in(-\infty,+\infty]$ the element $1_{\mathrm{SS}(\mu)} \in \mathrm{H}(\mathcal{C})_{\Phi}$ is invertible, and the automorphism

$$
\operatorname{Ad}_{1_{\mathrm{SS}(\mu)}}: \mathrm{H}(\mathcal{C})_{\Phi} \rightarrow \mathrm{H}(\mathcal{C})_{\Phi}
$$

preserves the subring of regular elements. The induced Poisson automorphism of $\mathrm{H}_{\mathrm{sc}}(\mathcal{C})_{\Phi}$ is given by

$$
\operatorname{Ad}_{1_{\mathrm{SS}(\mu)}}=\exp \left\{\eta_{\mu},-\right\} .
$$

Proof. That $1_{\mathrm{SS}(\mu)}$ is invertible follows from Lemma 5.3. The identity

$$
\operatorname{Ad}_{\exp (x)}=\exp \left(\operatorname{ad}_{x}\right)
$$

shows that

$$
\operatorname{Ad}_{1_{\mathrm{SS}(\mu)}}=\exp \left(\operatorname{ad}_{\epsilon_{\mu}}\right)=\exp \left(\operatorname{ad}_{(\mathbb{L}-1)^{-1} \eta_{\mu}}\right) .
$$

Theorem 6.3 shows that $\eta_{\mu}$ is regular, and since by [3, Theorem 5.1] the multiplication on $\mathrm{H}_{\text {reg }}(\mathcal{A})_{\Phi}$ is commutative modulo $(\mathbb{L}-1)$, it follows that this operation preserves $\mathrm{H}_{\mathrm{reg}}(\mathcal{A})_{\Phi}$. The last statement follows from the definition of the Poisson bracket on $\mathrm{H}_{\mathrm{sc}}(\mathcal{A})$.

6.4. The DT/PT correspondence. Consider the part of the Hilbert scheme Hilb $_{M, 0}$ parameterizing zero-dimensional subschemes of $M$. The morphism

$$
q: \operatorname{Hilb}_{M, 0} \rightarrow \mathcal{C}
$$

is $\Phi$-finite and so defines an element $\mathcal{H}_{0} \in \mathrm{H}(\mathcal{C})_{\Phi}$. As in Lemma 5.5 one has

$$
I_{\Phi}\left(\mathcal{H}_{0}\right)=\sum_{(\beta, n) \in \Delta}(-1)^{n} \mathrm{DT}(0, n) q^{n}=\mathrm{DT}_{0}(-q),
$$

where $q=x^{(0,1)} \in \mathbb{C}[\Delta]$.

Proposition 6.5. There is an identity

$$
\mathcal{H}_{\leqslant 1} * 1_{\mathcal{P}}=\mathcal{H}_{0} * 1_{\mathcal{P}} * \mathcal{H}_{\leqslant 1}^{\sharp}
$$

in $\mathrm{H}(\mathcal{C})_{\Phi}$.

Proof. We work in the ring $\mathrm{H}(\mathcal{C})_{\Phi}$ throughout. The first claim is that

$$
\mathcal{H}_{\leqslant 1} * 1_{\mathrm{SS}([\mu, \infty])}-1_{\mathrm{SS}([\mu, \infty])}^{\mathcal{O}} \rightarrow 0 \quad \text { as } \mu \rightarrow-\infty .
$$

To see this, fix $(\beta, m) \in \Delta$ and consider classes $(\beta, n)$ with $n<m$. There are only finitely many decompositions of such elements

$$
(\beta, n)=\left(\beta_{1}, n_{1}\right)+\left(\beta_{2}, n_{2}\right)
$$

such that the elements

$$
\pi_{\left(\beta_{1}, n_{1}\right)}\left(\mathcal{H}_{\leqslant 1}\right), \quad \pi_{\left(\beta_{2}, n_{2}\right)}\left(1_{\mathrm{SS}([\mu, \infty])}\right)
$$

are nonzero. By boundedness of the Hilbert scheme we can assume that $\mu$ is small enough so that for any of these, all points $\mathcal{O}_{M} \rightarrow A$ of $\operatorname{Hilb}_{M}\left(\beta_{1}, n_{1}\right)$ satisfy

$$
A \in \mathrm{SS}([\mu, \infty]) \text {. }
$$


Suppose given a diagram of sheaves on $M$ of the form

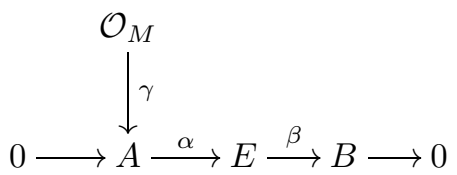

with $\gamma$ surjective and $[E]=(\beta, n)$. Then the above assumption on $\mu$ implies that

$$
B \in \mathrm{SS}([\mu, \infty]) \Longleftrightarrow E \in \mathrm{SS}([\mu, \infty]) .
$$

The argument of Lemma 4.3 then shows that

$$
\pi_{(\beta, n)}\left(\mathcal{H}_{\leqslant 1} * 1_{\mathrm{SS}([\mu, \infty])}\right)=\pi_{(\beta, n)}\left(1_{\mathrm{SS}([\mu, \infty])}^{\mathcal{O}}\right),
$$

which proves the claim. The same argument, but using Lemma 4.4, gives

$$
\mathcal{H}_{\leqslant 1}^{\sharp} * 1_{\mathrm{SS}}([\mu, \infty))-1_{\mathrm{SS}([\mu, \infty))}^{\mathcal{O}} \rightarrow 0 \quad \text { as } \mu \rightarrow-\infty .
$$

Since $\mathcal{P}=\mathrm{SS}(\infty)$, the proofs of Lemma 4.1 and Lemma 4.2 easily extend to give identities

$$
1_{\mathrm{SS}([\mu, \infty])}=1_{\mathcal{P}} * 1_{\mathrm{SS}([\mu, \infty))}, \quad 1_{\mathrm{SS}([\mu, \infty])}^{\mathcal{O}}=1_{\mathcal{P}}^{\mathcal{O}} * 1_{\mathrm{SS}([\mu, \infty))}^{\mathcal{O}} .
$$

Expression (37) can thus be rewritten

$$
\mathcal{H}_{\leqslant 1} * 1_{\mathcal{P}} * 1_{\mathrm{SS}}([\mu, \infty))-1_{\mathcal{P}}^{\mathcal{O}} * 1_{\mathrm{SS}([\mu, \infty))}^{\mathcal{O}} \rightarrow 0 \quad \text { as } \mu \rightarrow-\infty .
$$

The argument of Lemma 4.3 gives an identity

$$
1_{\mathcal{P}}^{\mathcal{O}}=\mathcal{H}_{0} * 1_{\mathcal{P}} .
$$

Multiplying (39) on the left by $1_{\mathcal{P}}^{\mathcal{O}}$ and using (40) give

$$
\mathcal{H}_{0} * 1_{\mathcal{P}} * \mathcal{H}_{\leqslant 1}^{\sharp} * 1_{\mathrm{SS}}([\mu, \infty))-1_{\mathcal{P}}^{\mathcal{O}} * 1_{\mathrm{SS}}^{\mathcal{O}}([\mu, \infty)) \rightarrow 0 \quad \text { as } \mu \rightarrow-\infty .
$$

Thus

$$
\mathcal{H}_{\leqslant 1} * 1_{\mathcal{P}} * 1_{\mathrm{SS}([\mu, \infty))}-\mathcal{H}_{0} * 1_{\mathcal{P}} * \mathcal{H}_{\leqslant 1}^{\sharp} * 1_{\mathrm{SS}([\mu, \infty))} \rightarrow 0 \quad \text { as } \mu \rightarrow-\infty .
$$

By Lemma 5.3 the element $1_{\mathrm{SS}}([\mu, \infty))$ is invertible so we can cancel it and deduce the result.

We can now prove the first part of Theorem 1.1 By Theorem 6.3

$$
1_{\mathcal{P}}=1_{\mathrm{SS}(\infty)}=\exp \left(\epsilon_{\infty}\right)
$$

where $\epsilon_{\infty} \in(\mathbb{L}-1)^{-1} \mathrm{H}_{\mathrm{reg}}(\mathcal{C})_{\Phi}$. The other elements in the identity of Proposition 6.5 lie in $\mathrm{H}_{\mathrm{reg}}(\mathcal{C})_{\Phi}$. Thus, using Corollary 6.4. we have an equation in $\mathrm{H}_{\mathrm{sc}}(\mathcal{C})_{\Phi}$

$$
\mathcal{H}_{\leqslant 1}=\mathcal{H}_{0} \cdot \exp \left(\left\{\eta_{\infty},-\right\}\right)\left(\mathcal{H}_{\leqslant 1}^{\sharp}\right) .
$$

Applying the integration map, the Poisson bracket vanishes, so we obtain

$$
I_{\Phi}\left(\mathcal{H}_{\leqslant 1}\right)=I_{\Phi}\left(\mathcal{H}_{0}\right) \cdot I_{\Phi}\left(\mathcal{H}_{\leqslant 1}^{\sharp}\right),
$$

and the result follows from (28), (29), and (36). 


\section{RATiONALity}

In this section we complete the proof of Theorem 1.1 by proving the rationality statement of part (b).

7.1. DT invariants counting semistables. Recall the elements $\eta_{\mu}$ of Theorem 6.3 and decompose them as

$$
\eta_{\mu}=\sum_{\mu(\gamma)=\mu} \eta_{\gamma}
$$

Following [16, Section 6.4], we can define invariants $N_{(\beta, n)} \in \mathbb{Q}$ counting semistable sheaves of Chern character $(\beta, n) \in \Delta$ by setting

$$
I\left(\eta_{(\beta, n)}\right)=-N_{(\beta, n)} x^{(\beta, n)} .
$$

Most of the following result was obtained previously by Joyce and Song [16, Theorem 6.16]; since the proof is easy we include it here.

Lemma 7.1. Assume $\beta \in N_{1}(M)$ is effective and nonzero. Then the invariants $N_{(\beta, n)}$ satisfy

(a) $N_{(\beta, n)}=N_{(\beta, n+\beta \cdot H)}$,

(b) $N_{(\beta, n)}=N_{(\beta,-n)}$

and are independent of the choice of ample divisor $H$.

Proof. Part (a) holds because tensoring with $\mathcal{O}_{M}(H)$ defines an automorphism of the stack $\mathcal{C}$ taking semistable sheaves of class $(\beta, n)$ to semistable sheaves of class $(\beta, n+\beta \cdot H)$. Part (b) follows in the same way using the dualizing functor $\mathbb{D}$ and Lemma 6.1. The independence of the choice of ample divisor $H$ follows from Proposition 7.2 below, since the subcategory $\mathrm{SS}(I)$ corresponding to the interval $I=\mathbf{R}_{>0} \subset \mathbf{R}$ consists of those objects of $\mathcal{Q} \cap \mathcal{C}$ whose quotients all have positive Euler characteristic and is therefore independent of the choice of the ample divisor $H$.

Given a $\Delta$-graded $K(\operatorname{Var} / \mathbb{C})\left[\mathbb{L}^{-1}\right]$-algebra

$$
A=\bigoplus_{\gamma \in \Delta} A_{\gamma},
$$

we define an automorphism $\chi$ of $A$, whose action we write as $a \mapsto a^{\chi}$, by the rule

$$
a^{\chi}=\mathbb{L}^{\chi(\gamma)} \cdot a \text { for } a \in A_{\gamma} .
$$

Here $\chi(\gamma)$ is the Euler characteristic of a sheaf of class $\gamma$.

We can consider the automorphism $\chi$ as defining an action of $\mathbb{Z}$ on $A$ and form the skew-group algebra $\hat{A}=A * \mathbb{Z}$. In terms of generators and relations

$$
\hat{A}=A\left\langle y, y^{-1}\right\rangle /\left(y * a-a^{\chi} * y\right) .
$$

Suppose the quotient algebra

$$
A_{\mathrm{sc}}=A /(\mathbb{L}-1) A
$$

is commutative, and let $\{-,-\}$ be the induced Poisson bracket. Then the quotient

$$
\hat{A}_{\mathrm{sc}}=\hat{A} /(\mathbb{L}-1) \hat{A}
$$

is a commutative algebra, isomorphic to $A_{\mathrm{sc}}\left[y, y^{-1}\right]$, and the induced Poisson bracket satisfies

$$
\{y, a\}=\chi(\gamma) \cdot y a \quad \text { for } a \in A_{\gamma}
$$


All this extends in the obvious way to the topologically graded algebras $A_{\Phi}$.

Proposition 7.2. Given an interval $I \subset(-\infty,+\infty]$ bounded below, the element

$$
1_{\mathrm{SS}(I)}^{-1} * 1_{\mathrm{SS}(I)}^{\chi} \in \mathrm{H}(\mathcal{C})_{\Phi}
$$

is regular and

$$
I_{\Phi}\left(1_{\mathrm{SS}(I)}^{-1} * 1_{\mathrm{SS}(I)}^{\chi}\right)=\exp \left(\sum_{\substack{(\beta, n) \in \Delta \\ \mu(\beta, n) \in I}} n N_{(\beta, n)} x^{(\beta, n)}\right) \in \mathbb{C}[\Delta]_{\Phi} .
$$

Proof. By definition of the product in the algebra $\hat{\mathrm{H}}(\mathcal{C})_{\Phi}$ one has

$$
\operatorname{Ad}_{1_{\mathrm{SS}(I)}^{-1}}^{-1}(y)=1_{\mathrm{SS}(I)}^{-1} * y * 1_{\mathrm{SS}(I)}=1_{\mathrm{SS}(I)}^{-1} * 1_{\mathrm{SS}(I)}^{\chi} * y .
$$

The first claim then follows by the argument of Corollary 6.4, Lemma 6.2 and Corollary 6.4 imply that

$$
\operatorname{Ad}_{1_{\mathrm{SS}(I)}^{-1}}^{-1} \prod_{\mu \in I} \operatorname{Ad}_{1_{\mathrm{SS}(\mu)}^{-1}}^{-1}=\prod_{\mu \in I} \exp \left\{-\eta_{\mu},-\right\}: \hat{\mathrm{H}}_{\mathrm{sc}}(\mathcal{C})_{\Phi} \longrightarrow \hat{\mathrm{H}}_{\mathrm{sc}}(\mathcal{C})_{\Phi}
$$

where the product of endomorphisms are taken in order of ascending slope and the infinite products are interpreted as in Lemma 6.2. The homomorphism of topologically $\Delta$-graded Poisson algebras $I_{\Phi}$ induces a Poisson algebra map

$$
I_{\Phi}: \hat{\mathrm{H}}_{\mathrm{sc}}(\mathcal{C})_{\Phi} \longrightarrow \hat{\mathbb{C}}[\Delta]_{\Phi}
$$

Applying this gives

$$
I_{\Phi}\left(1_{\mathrm{SS}(I)}^{-1} * 1_{\mathrm{SS}(I)}^{\chi}\right) \cdot y=\prod_{\mu \in I} \exp \left\{-I_{\Phi}\left(\eta_{\mu}\right),-\right\}(y) .
$$

By Lemma 7.3 and (41) this gives

$$
I_{\Phi}\left(1_{\mathrm{SS}(I)}^{-1} * 1_{\mathrm{SS}(I)}^{\chi}\right) \cdot y=\prod_{\substack{(\beta, n) \in \Delta \\ \mu(\beta, n) \in I}} \exp \left(n N_{(\beta, n)} x^{(\beta, n)}\right) \cdot y
$$

and the result follows.

We used the following easy result.

Lemma 7.3. Suppose $A$ is a topological Poisson algebra and $a, b \in A$ satisfy

$$
\{a, b\}=n a b .
$$

Then, provided the relevant sums converge,

$$
\exp \{a,-\}(b)=\exp (n a) \cdot b .
$$

Furthermore, if $n=0$, then the automorphisms $\exp \{a,-\}$ and $\exp \{b,-\}$ commute and

$$
\exp \{a+b,-\}=\exp \{a,-\} \circ \exp \{b,-\} .
$$

Proof. This is left to the reader. 
7.2. Rationality. Recall the definition of the Laurent series

$$
\mathrm{PT}_{\beta}(q)=\sum_{n \in \mathbb{Z}} \mathrm{PT}(\beta, n) q^{n}
$$

from the introduction. To prove Theorem 1.1(b), we follow a strategy of Toda and prove more, namely

Theorem 7.4. There is an identity in $\mathbb{C}[\Delta]_{\Phi}$

$$
\sum_{\beta \geqslant 0} \operatorname{PT}_{\beta}(-q) x^{\beta}=\exp \left(\sum_{\substack{\beta \geqslant 0 \\ n \geqslant 0}} n N_{(\beta, n)} x^{\beta} q^{n}\right) \cdot \sum_{\beta \geqslant 0} L_{\beta}(q) x^{\beta}
$$

where for each effective class $\beta \in N_{1}(M)$ the expression $L_{\beta}(q)$ is a Laurent polynomial in $q$ invariant under $q \leftrightarrow q^{-1}$.

This is enough by Lemma 7.1 and the following simple result.

Lemma 7.5. Fix a positive integer $d \geqslant 1$. Suppose given rational numbers $a_{n} \in \mathbb{Q}$ such that for all $n \in \mathbb{Z}$ one has

$$
a_{n}=a_{-n}, \quad a_{n+d}=a_{n} .
$$

Then the power series

$$
\sum_{n \geqslant 0} n a_{n} q^{n}
$$

is the Taylor expansion of a rational function in $q$ invariant under $q \leftrightarrow q^{-1}$.

Proof. This is a simple calculation; see [27, Lemma 4.6].

Given $\mu>0$, let us consider the following tautology in $\mathrm{H}(\mathcal{C})_{\Phi}$ :

$$
1_{\mathrm{SS}([0, \mu])}^{-1} * \mathcal{H}_{\leqslant 1}^{\sharp} * 1_{\mathrm{SS}([0, \mu])}=\left(1_{\mathrm{SS}([0, \mu])}^{-1} * 1_{\mathrm{SS}([0, \mu])}^{\chi}\right) * \mathcal{G}_{\mu}
$$

where

$$
\mathcal{G}_{\mu}=\left(1_{\mathrm{SS}([0, \mu])}^{\chi}\right)^{-1} * \mathcal{H}_{\leqslant 1}^{\sharp} * 1_{\mathrm{SS}([0, \mu])} .
$$

Note that by Lemma 6.2 and Corollary 6.4 the left-hand side of (43) is regular.

Since the Poisson bracket on $\mathbb{C}[\Delta]_{\Phi}$ is zero, it follows from (29) that

$$
I_{\Phi}\left(1_{\mathrm{SS}([0, \mu])}^{-1} * \mathcal{H}_{\leqslant 1}^{\sharp} * 1_{\mathrm{SS}([0, \mu])}\right)=I_{\Phi}\left(\mathcal{H}_{\leqslant 1}^{\sharp}\right)=\sum_{\beta \geqslant 0} \operatorname{PT}_{\beta}(-q) x^{\beta} .
$$

By Proposition 7.2 the first term on the right-hand side is also regular, and

$$
I_{\Phi}\left(1_{\mathrm{SS}([0, \mu])}^{-1} * 1_{\mathrm{SS}([0, \mu])}^{\chi}\right)=\exp \left(\sum_{\substack{(\beta, n) \in \Delta \\ \mu(\beta, n) \in[0, \mu]}} n N_{(\beta, n)} x^{(\beta, n)}\right) .
$$

As $\mu \rightarrow \infty$, this tends to the first term in Toda's identity. The following result therefore completes the proof of Theorem 1.1

Proposition 7.6. The element $\mathcal{G}_{\mu}$ is regular and

$$
\lim _{\mu \rightarrow \infty} I_{\Phi}\left(\mathcal{G}_{\mu}\right)=\sum_{\beta \geqslant 0} L_{\beta}(q) x^{\beta},
$$

where each $L_{\beta}(q)$ is a Laurent polynomial, invariant under $q \leftrightarrow q^{-1}$. 
Proof. Recall the subalgebra $\mathrm{H}(\mathcal{Q} \cap \mathcal{C}) \subset \mathrm{H}(\mathcal{C})$ and its anti-involution $\mathbb{D}$ defined in Section 5.4. Consider the composition

$$
R=\chi \circ \mathbb{D} \text {. }
$$

Since $\chi(\mathbb{D}(E))=-\chi(E)$ for any sheaf $E \in \mathcal{Q} \cap \mathcal{C}$, it follows that $R$ is also an anti-involution. There is an obvious involution of $\mathbb{C}[\Delta]$, which we also denote by $R$, defined by

$$
R\left(x^{(\beta, n)}\right)=x^{(\beta,-n)} .
$$

Note that by (32), for any $a \in \mathrm{H}(\mathcal{Q} \cap \mathcal{C})$ one has

$$
I(R(a))=R(I(a)) .
$$

We claim that the involution $R$ fixes the element

$$
1_{\mathrm{SS}([-\mu, \mu])}^{\mathcal{O}} \in \mathrm{H}(\mathcal{Q} \cap \mathcal{C})_{\Phi}
$$

In fact, for all $\gamma \in \Delta$

$$
R\left(\pi_{\gamma}\left(1_{\mathrm{SS}([-\mu, \mu])}^{\mathcal{O}}\right)\right)=\pi_{R(\gamma)}\left(1_{\mathrm{SS}([-\mu, \mu])}^{\mathcal{O}}\right) \in \mathrm{H}(\mathcal{Q} \cap \mathcal{C}) .
$$

To see this, note first that if $E \in \mathcal{Q} \cap \mathcal{C}$ has class $\gamma=(\beta, n)$, then by Serre duality

$$
H^{0}(M, \mathbb{D}(E)) \cong H^{1}(M, E)^{*},
$$

and so

$$
n=\chi(E)=\operatorname{dim} H^{0}(M, E)-\operatorname{dim} H^{0}(M, \mathbb{D}(E)) .
$$

Take a finite stratification

$$
\mathrm{SS}([-\mu, \mu])_{\gamma}=\coprod_{r \geqslant 0} \mathrm{SS}([-\mu, \mu])_{\gamma} \cap \mathcal{M}_{r}
$$

as in Lemma 2.5. It follows from Lemma 6.1 and (48) that

$$
\mathbb{D}\left(\mathrm{SS}([-\mu, \mu])_{(\beta, n)} \cap \mathcal{M}_{r}\right)=\mathrm{SS}([-\mu, \mu])_{(\beta,-n)} \cap \mathcal{M}_{r-n} .
$$

Applying Lemma 2.5.

$$
\mathbb{D}\left(1_{\mathrm{SS}([-\mu, \mu])_{(\beta, n)}^{\mathcal{O}} \cap \mathcal{M}_{r}}\right)=\mathbb{L}^{n} \cdot 1_{\mathrm{SS}([-\mu, \mu])_{(\beta,-n)}^{\mathcal{O}} \cap \mathcal{M}_{r-n}}^{\mathcal{O}},
$$

and the claim follows.

Next define an element

$$
\mathcal{J}_{\mu}=\mathcal{H}_{\leqslant 1}^{\sharp} * 1_{\mathrm{SS}([-\mu, \mu])} \in \mathrm{H}(\mathcal{C})_{\Phi} .
$$

By the argument we used to prove (39)

$$
\mathcal{J}_{\mu}-1_{\mathrm{SS}([-\mu, \mu])}^{\mathcal{O}} \rightarrow 0 \quad \text { as } \mu \rightarrow \infty .
$$

Lemma 6.2 implies that there is an identity

$$
1_{\mathrm{SS}([-\mu, \mu])}=1_{\mathrm{SS}([0, \mu])} * 1_{\mathrm{SS}([-\mu, 0))}
$$

in $\mathrm{H}(\mathcal{C})_{\Phi}$. Thus

$$
\mathcal{G}_{\mu}=\left(1_{\mathrm{SS}([0, \mu])}^{\chi}\right)^{-1} * \mathcal{J}_{\mu} * 1_{\mathrm{SS}([-\mu, 0))}^{-1} .
$$

Let us also consider the element

$$
\mathcal{G}_{\mu}^{\prime}=\left(1_{\mathrm{SS}((0, \mu])}^{\chi}\right)^{-1} * \mathcal{J}_{\mu} * 1_{\mathrm{SS}([-\mu, 0])}^{-1} .
$$


We can modify equation (43) by replacing the closed intervals by intervals open at 0 . This has the effect of replacing the element $\mathcal{G}_{\mu}$ by $\mathcal{G}_{\mu}^{\prime}$. Equations (44) and (45) are unchanged by this modification, which shows that

$$
I_{\Phi}\left(\mathcal{G}_{\mu}\right)=I_{\Phi}\left(\mathcal{G}_{\mu}^{\prime}\right) \text {. }
$$

Using Lemma 6.1 and the fact that $R$ is an anti-automorphism, we find

$$
R\left(\mathcal{G}_{\mu}^{\prime}\right)=\left(1_{\mathrm{SS}([0, \mu])}^{\chi}\right)^{-1} * R\left(\mathcal{J}_{\mu}\right) * 1_{\mathrm{SS}([-\mu, 0))}^{-1} .
$$

It follows from (47) and (49) that

$$
\mathcal{G}_{\mu}-R\left(\mathcal{G}_{\mu}^{\prime}\right)=\left(1_{\mathrm{SS}([0, \mu])}^{\chi}\right)^{-1} *\left(\mathcal{J}_{\mu}-R\left(\mathcal{J}_{\mu}\right)\right) * 1_{\mathrm{SS}([-\mu, 0))}^{-1} \rightarrow 0
$$

as $\mu \rightarrow \infty$. Thus by (46)

$$
I_{\Phi}\left(\mathcal{G}_{\mu}\right)-R\left(I_{\Phi}\left(\mathcal{G}_{\mu}\right)\right) \rightarrow 0
$$

On the other hand, the limit of $I_{\Phi}\left(\mathcal{G}_{\mu}\right)$ as $\mu \rightarrow \infty$ certainly exists because the other factors in (43) have convergent integrals. Thus we conclude that this limit is invariant under $R$ and the result follows.

\section{ACKNOWLEDGEMENTS}

Thanks most of all to Dominic Joyce who patiently explained many things about motivic Hall algebras. In particular he pointed out how to apply his virtual indecomposable technology to replace the Kontsevich-Soibelman integration map used in an earlier verion of this paper. The author is also very grateful to Yukinobu Toda who explained his approach to Theorem 1.1 at the inaugural meeting of the Homological Research Seminar (HoRSe) and to Richard Thomas who provided a lot of encouragement and technical know-how. Finally, the author is very happy to acknowledge the entertaining and useful conversations he has enjoyed with Davesh Maulik, Rahul Pandharipande, and Balázs Szendröi.

\section{REFERENCES}

[1] K. Behrend, Donaldson-Thomas type invariants via microlocal geometry, Ann. of Math. (2) 170 (2009), no. 3, 1307-1338. MR2600874 (2011d:14098)

[2] K. Behrend and B. Fantechi, Symmetric obstruction theories and Hilbert schemes of points on threefolds, Algebra Number Theory 2 (2008), no. 3, 313-345. MR2407118 (2009c:14006)

[3] T. Bridgeland, An introduction to motivic Hall algebras, preprint arXiv 1002.4372.

[4] T. Bridgeland and A. Maciocia, Fourier-Mukai transforms for elliptic and K3 fibrations, J. Algebraic Geom. 11 (2002), no. 4, 629-657. MR1910263 (2004e:14019)

[5] J. Engel and M. Reineke, Smooth models of quiver moduli, Math. Z. 262 (2009), no. 4, 817-848. MR2511752(2010h:16033)

[6] W. Fulton, Intersection Theory, 2nd edition, Springer-Verlag, Berlin, 1998. xiv+470 pp. MR:1644323 (99d:14003)

[7] A. Grothendieck, Techniques de construction et théorèmes d'existence en géométrie algébrique IV: les schémas d'Hilbert, Séminaire Bourbaki, Vol. 6, Soc. Math. de France, Paris, 1995, Exp. no. 221, 249-276. MR 1611822

[8] A. Grothendieck, Élements de géométrie algébrique (rédigés avec la collaboration de Jean Dieudonné): IV. Étude locale des schémas et des morphismes de schémas, Troisième partie. Publications Mathématiques de l'IHÉS 28 (1966), 5-255. MR0217086 (36:178)

[9] D. Happel, I. Reiten, and S. Smalo, Tilting in abelian categories and quasitilted algebras, Mem. Amer. Math. Soc. 120 (1996), no. 575, viii+ 88 pp. MR1327209 (97j:16009)

[10] D. Huybrechts and M. Lehn, The geometry of moduli spaces of shaves, Aspects of Mathematics, E31, 1997. xiv+269 pp. MR1450870 (98g:14012)

[11] D. Joyce, Motivic invariants of Artin stacks and stack functions, Q. J. Math. 58 (2007), no. 3, 345-392. MR2354923 (2010b:14004) 
[12] D. Joyce, Configurations in abelian categories. I. Basic properties and moduli stacks, Adv. Math. 203 (2006), no. 1, 194-255. MR2231046 (2007b:14023)

[13] D. Joyce, Configurations in abelian categories. II. Ringel-Hall algebras, Adv. Math. 210 (2007), no. 2, 635-706. MR2303235 (2008f:14022)

[14] D. Joyce, Configurations in abelian categories. III. Stability conditions and identities, Adv. in Math. 215 (2007), no. 1, 153-219. MR2354988 (2009d:18015a)

[15] D. Joyce, Configurations in abelian categories. IV. Invariants and changing stability conditions, Adv. in Math. 217 (2008), no. 1, 125-204. MR2357325 (2009d:18015b)

[16] D. Joyce and Y. Song, A theory of generalized DT invariants, preprint arXiv:0810.5645.

[17] J. Kollár and S. Mori, Birational geometry of algebraic varieties, Cambridge University Press, Cambridge, 1998. viii+254 pp. MR.1658959 (2000b:14018)

[18] M. Kontsevich and Y. Soibelman, Stability structures, motivic Donaldson-Thomas invariants and cluster transformations, preprint arxiv 0811.2435.

[19] M. Levine and R. Pandharipande, Algebraic cobordism revisited, Invent. Math. 176 (2009), no. 1, 63-130. MR2485880 (2010h:14033)

[20] J. Li, Zero dimensional Donaldson-Thomas invariants of threefolds, Geom. Topology 10 (2006), 2117-2171. MR2284053 (2007k:14116)

[21] D. Maulik, N. Nekrasov, A. Okounkov, R. Pandharipande, Gromov-Witten theory and Donaldson-Thomas theory. I. Compos. Math. 142 (2006), no. 5, 1263-1285. MR2264664 (2007i:14061)

[22] N. Nitsure, Construction of Hilbert and Quot schemes, Fundamental algebraic geometry, 105-137, Math. Surveys Monogr., 123, Amer. Math. Soc., Providence, RI, 2005. MR2223407

[23] R. Pandharipande and R. P. Thomas, Curve counting via stable pairs in the derived category, Invent. Math. 178 (2009), no. 2, 407-447. MR2545686 (2010h:14089)

[24] M. Reineke, The Harder-Narasimhan system in quantum groups and cohomology of quiver moduli, Invent. Math. 152 (2003), no. 2, 349-368. MR1974891 (2004a:16028)

[25] J. Stoppa and R. P. Thomas, Hilbert schemes and stable pairs: GIT and derived category wall crossings, preprint.

[26] R. P. Thomas, A holomorphic Casson invariant for Calabi-Yau 3-folds, and bundles on $K 3$ fibrations, J. Differential Geom. 54 (2000), no. 2, 367-438. MR1818182 (2002b:14049)

[27] Y. Toda, Generating functions of stable pair invariants via wall-crossings in derived categories, New developments in algebraic geometry, integrable systems and mirror symmetry (RIMS, Kyoto, 2008), 389-434, Adv. Stud. Pure Math., 59, Math. Soc. Japan, Tokyo (2010). MR2683216

[28] Y. Toda, Curve counting theories via stable objects I. DT/PT correspondence, J. Amer. Math. Soc. 23 (2010), no. 4, 1119-1157. MR2669709

[29] A. Vistoli, Grothendieck topologies, fibered categories and descent theory. Fundamental algebraic geometry, 1-104, Math. Surveys Monogr., 123, Amer. Math. Soc., Providence, RI, 2005. MR.2223406

All Souls College, Oxford, OX1 4AL United Kingdom 